\title{
Petrogenesis of the Nanling Mountains granites from South China: Constraints from systematic apatite geochemistry and whole-rock geochemical and $\mathrm{Sr}-\mathrm{Nd}$ isotope compositions
}

\author{
Pei-Shan Hsieh ${ }^{\mathrm{a}}$, Cheng-Hong Chen ${ }^{\mathrm{a}, *}$, Huai-Jen Yang ${ }^{\mathrm{b}}$, Chi-Yu Lee ${ }^{\mathrm{a}}$ \\ ${ }^{a}$ Department of Geosciences, National Taiwan University, No. 1, Roosevelt Road Section 4, Taipei 106, Taiwan \\ ${ }^{\mathrm{b}}$ Department of Earth Sciences, National Cheng Kung University, Tainan, Taiwan
}

Received 1 August 2007; received in revised form 12 February 2008; accepted 18 February 2008

\begin{abstract}
The widespread Mesozoic granitoids in South China $\left(\sim 135,300 \mathrm{~km}^{2}\right)$ were emplaced in three main periods: Triassic $(16 \%$ of the total surface area of Mesozoic granitoids), Jurassic (47\%), and Cretaceous (37\%). Though much study has been conducted on the most abundant Jurassic Nanling Mountains (NLM) granites, their rock affinities relative to the Triassic Darongshan (DRS) and Cretaceous Fuzhou-Zhangzhou Complex (FZC) granites which are typical S- and I-type, respectively, and the issue of their petrogenetic evolution is still the subject of much debate. In this study, we discuss the petrogenesis of NLM granites using apatite geochemistry combined with wholerock geochemical and $\mathrm{Sr}-\mathrm{Nd}$ isotope compositions. Sixteen apatite samples from six granite batholiths, one gabbro, and three syenite bodies in the NLM area were analyzed for their major and trace element abundances and compared with those collected from DRS $(n=7)$ and FZC $(n=6)$ granites. The apatite geochemistry reveals that $\mathrm{Na}, \mathrm{Si}, \mathrm{S}, \mathrm{Mn}, \mathrm{Sr}, \mathrm{U}$, Th concentrations and REE distribution patterns for apatites from DRS and FZC granites basically are similar to the S and I granite types of the Lachlan Fold Belt (Australia), whereas those from NLM granites have intermediate properties and cannot be correlated directly with these granite types. According to some indications set by the apatite geochemistry (e.g., lower U and higher Eu abundances), NLM apatites appear to have formed under oxidizing conditions. In addition, we further found that their REE distribution patterns are closely related to aluminum saturation index (ASI) and $\mathrm{Nd}$ isotope composition, rather than $\mathrm{SiO}_{2}$ content or degree of differentiation, of the host rock. The majority of apatites from NLM granites (ASI $=0.97-1.08$ and $\varepsilon \mathrm{Nd}(T)=-8.8$ to -11.6$)$ display slightly right-inclined apatite REE patterns distinguishable from the typical S- and I-type. However, those from few granites with ASI $>1.1$ and $\varepsilon \mathrm{Nd}(T)<-11.6$ have REE distribution patterns (nearflat) similar to DRS apatites whereas those from granites with ASI $<1.0$ and $\varepsilon \mathrm{Nd}(T)>-6.6$ and gabbro and syenite are similar to FZC apatites (strongly right-inclined). In light of $\mathrm{Sr}$ and $\mathrm{Nd}$ isotope compositions, magmas of NLM intrusives, except gabbro and syenite, and few granites with $\varepsilon \operatorname{Nd}(T)>-8$, generally do not involve a mantle component. Instead, they fit with a melt derived largely from in situ melting or anatexis of the pre-Mesozoic (mainly Caledonian) granitic crust with subordinate pre-Yanshanian (mainly Indosinian) granitic crust. We suggest that an application, using combined whole-rock ASI and $\varepsilon \mathrm{Nd}(T)$ values, is as useful as the apatite geochemistry for recognizing possible sources for the NLM granites.
\end{abstract}

(C) 2008 Elsevier Ltd. All rights reserved.

Keywords: Apatite geochemistry; ASI (aluminum saturation index); Mesozoic granitoids; Nanling Mountains; South China

\section{Introduction}

South China is composed of Yangtze Block in the northwest and Cathaysia Block in the southeast (Fig. 1a), and the

\footnotetext{
${ }^{*}$ Corresponding author. Tel.: +8862 33665872; fax: +886223636095.

E-mail address: chench@ntu.edu.tw (C.-H. Chen).
}

latter is characterized by record of repeated granitic magmatism since the Neoproterozoic. Mesozoic igneous rocks are the most widely exposed in the Cathaysia Block (approximately $50 \%$ of the total surface area) and many are enriched in $\mathrm{Sn}, \mathrm{W}, \mathrm{Bi}, \mathrm{Mo}, \mathrm{Pb}, \mathrm{Zn}$, and $\mathrm{Cu}$ ore deposits (GRGNP, 1989). Three main episodes of the Mesozoic magmatic events have occurred; Triassic, Jurassic, and Cretaceous, 

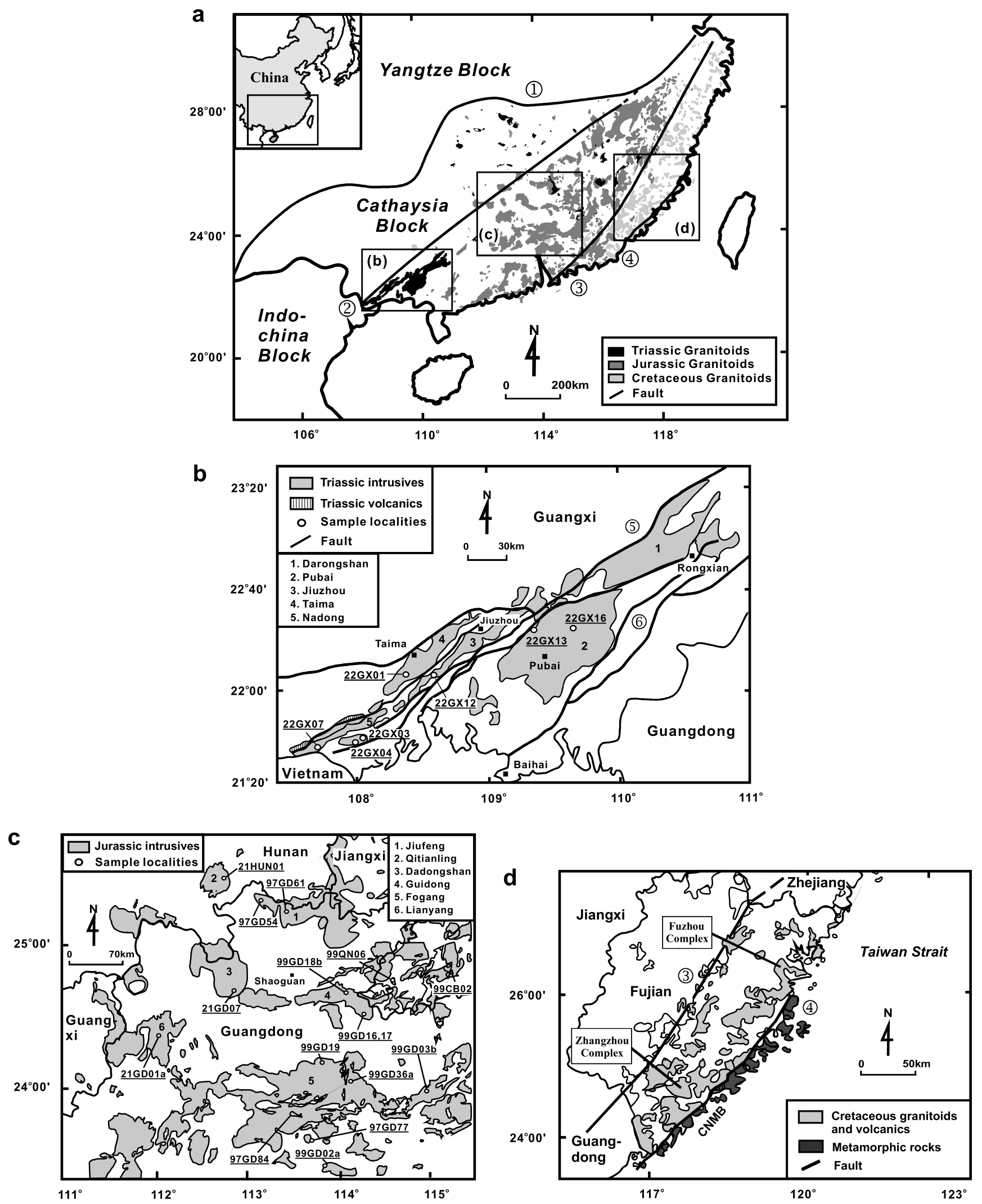

Fig. 1. (a) Simplified geological map of the Mesozoic granitoids in S. China, with sample localities of (b) Indosinian (Triassic) Darongshan (DRS) granitic suites (modified after Deng et al., 2004), (c) Early Yanshanian (Jurassic) Nanling Mountains (NLM) batholiths and plutons, and (d) Late Yanshanian (Cretaceous) Fuzhou-Zhangzhou Complex (FZC) granitic plutons (Fuzhou, Dayang, and Liangjiang plutons of the Fuzhou Complex and Zudi, Yanqian, and Changtai plutons of the Zhangzhou Complex) (modified after Chen et al., 2000). Major faults in S. China include (1) Jiangshan-Shaoxing, DongxiangPingxiang, and Xupu-Sanjiang deep fault zone; (2) Shi-Hang zone; (3) Zhenghe-Dapu deep fault zone; (4) Changle-Nanao deep fault zone; (5) Bobai-Cenxi deep fault zone; and (6) Lingshan-Tangxian deep fault zone. 
and they are conventionally referred as the Indosinian, Early Yanshanian (EY), and Late Yanshanian (LY) stages of orogeny (Zhou and Li, 2000; Li et al., 2004). Lithologically, granites and rhyolites $(>95 \%)$ predominate over basic and intermediate rocks, and volumetrically, Indosinian granitoids crop out in a total area of $\sim 20,900 \mathrm{~km}^{2}$ ( $16 \%$ of the total surface area of Mesozoic granitoids), EY granitoids $\sim 64,100 \mathrm{~km}^{2}(47 \%)$, and LY granitoids $\sim 50,300 \mathrm{~km}^{2}$ (37\%) (Sun, 2006). For more than half a century, the origin and petrogenesis of extensive EY granitoids and the process of related mineralization have stimulated the interest of geologists. Moreover, whether the EY magmatism caused the extensive crustal growth or whether the crustal evolution is related to the geodynamic environment of the Mesozoic South China continent is still hotly debated (Gilder et al., 1996; Chen and Jahn, 1998; Zhou and Li, 2000; Pirajno and Bagas, 2002; Chen and Grapes, 2003; Li et al., 2003; $\mathrm{Xu}$ et al., 2005; $\mathrm{Li}$ and $\mathrm{Li}, 2007$ ). To better constrain mantle input to the crust, an important step is to understand the petrogenesis of EY granitoids.

Since Chappell and White (1974) first proposed the concept of two contrasting granite types ( $\mathrm{S}$ and I) based on the distinction of chemical and mineralogical compositions, granites have commonly been related to derivation by partial melting of two different types of source material-sedimentary and igneous, and the derived granites can inherit the geochemical and isotopic characteristics from their source rocks. S-type granites are commonly thought to represent melting of "reworked" continental crust in contrast to I-type granites whose sources basically have not been subject to significant chemical weathering (Chappell and White, 2001). The term "granite" they used and subsequently in this study, in a broad sense, includes all plutonic rocks dominated by quartz and feldspar, and is analogous to "granitoid" (Streckeisen, 1976). However, to identify such two types of rock using the criteria proposed by Chappell and White (1974) is not always possible, particularly if mixedcharacteristics of source regions or multiple sources with magma mingling have occurred (Clemens, 2003). Therefore, different petrogenetic discrimination schemes, such as the iron oxide series (Ishihara, 1977), relative abundances of whole-rock trace elements (Pearce et al., 1984), combining field observations and petrographical, chemical, and isotopic criteria (Barbarin, 1999), and chemical discrimination of apatite from granites (Zhang et al., 1985; Sha and Chappell, 1999; Belousova et al., 2002), have been proposed.

It has long been suggested that the Mesozoic S-type granites in S. China are mainly distributed in the interior of the Cathaysia Block, and I-type granites only crop out near the coast (Jahn et al., 1976, 1990; Gilder et al., 1996; Chen and Jahn, 1998; Pirajno and Bagas, 2002). However, the origin of the voluminous EY granitoids in the center of this Block is still under debate because of the complexity of these rocks. For example, the largest Fogang batholith $\left(\sim 6000 \mathrm{~km}^{2}\right)$ is thought to include the $\mathrm{S}-$, I-, and A-type granites based on mineralogical, wholerock geochemical and $\mathrm{Sr}-\mathrm{Nd}$ isotopic criteria ( $\mathrm{Li}$ et al.,
2007 and references therein). Any petrogenetic model developed to account for magma generation of such a large and complicated batholith needs to address the regional control for the distribution of magmatism, rather than relying on a single batholith alone. Furthermore, the use of effective geochemical and isotopic discrimination parameters is critical to understanding of the overall picture of the Mesozoic granitoids in S. China. As apatites in the meta- and peraluminous rocks appear to contain many elements that are sensitive to different physical conditions (Bea, 1996), apatite geochemistry is considered as an additional distinguishing criterion.

Apatite, with an ideal formula of $\mathrm{Ca}_{10}\left(\mathrm{PO}_{4}\right)_{6}(\mathrm{~F}, \mathrm{OH}$, $\mathrm{Cl}_{2}$, occurs as an accessory mineral in almost all kinds of rock. In igneous rocks, its abundance varies directly with the phosphorous content and inversely with the increasing silica content of the host rock (Frietsch and Perdahl, 1995). Apatite is also an excellent host of some trace elements such as REE, Sr, U, and Th in the natural system which are useful not only for understanding ore genesis (Treloar and Colley, 1996), but also in the field of mineral exploration (Belousova et al., 2002; Mordberg et al., 2006). With regard to petrogenetic applications, Sha and Chappell (1999) found that apatites can concentrate many minor and trace elements whose abundances and ratios are sensitive to factors controlling the fundamental differences between I- and S-type granites and suggested that the results have important implications for identifying different types of granites and potential significance for determining the provenance of sedimentary rocks.

The aims of this study are threefold. Since apatite geochemistry has been shown to be an effective parameter that correlates well with S- and I-type granites in the Lachlan Folded Belt (LFB), Australia (Sha and Chappell, 1999), it is used here for the first time to differentiate the wellestablished Mesozoic S- and I-type granites in S. China. On the basis of apatite geochemistry, many other granites in S. China, particularly those in the Nanling Mountains (NLM) area that show more complicated geochemical and isotopic characteristics than the simple S-I division, are examined. They are compared in order to reveal the conditions of magma crystallization, such as the redox state and the effect of coexisting accessory minerals. This may help to better constrain the potential for ore mineral exploration related to these granites (Belousova et al., 2002). Finally, correlations between the apatite geochemistry and the whole-rock parameters (major and trace element abundances and $\mathrm{Sr}$ and $\mathrm{Nd}$ isotope compositions) are investigated with regard to the NLM granites. These results help to provide better constraints on the petrogenetic model and tectonic setting for these granites.

\section{Geological background and sampling}

The Yangtze and Cathaysia Blocks are separated by a fault system including the Jiangshan-Shaoxing fault in Zhejiang province, the Dongxiang-Pingxiang fault in Jiangxi 
province, and the Xupu-Sanjiang in Guangxi province (Fig. 1a). Basement of the Cathaysia Block was Mesoproterozoic to Ordovician flysch sequences, possibly with a small Archean nucleus, before being subject to greenschist to amphibolite facies metamorphism (Li et al., 1991; Wang and Mo, 1995; Xu et al., 2005). It was unconformably covered by the Devonian to Triassic sediments and intruded by the wide spreading Mesozoic igneous rocks (Gilder et al., 1996; Chen and Jahn, 1998). Temporally, the Triassic rocks expose dispersively in the Cathaysia interior, whereas the Jurassic and Cretaceous rocks are more concentrated in the central and a narrow zone close to the SE coastal areas, respectively, of the Cathaysia Block (Fig. 1a).

Darongshan (DRS) granitic suites, including Darongshan, Pubai, Taima, Jiuzhou, and Nadong batholiths, are the most important intrusive rocks related to the Indosinian orogeny (Triassic) in the Cathaysia Block. They are distributed in an elongate fashion for $>400 \mathrm{~km}$ in the southeastern part of Guangxi Province, with a total area of exposure for granites over $10,000 \mathrm{~km}^{2}$ (Fig. 1b). During Late Permian to Triassic, this area was strongly folded, and intrusion of DRS granitic suites was controlled by the Bobai-Cenxi and Lingshan-Tangxian deep faults and their bifurcated faults (Deng et al., 2004). DRS granitic suites are mainly composed of cordierite-biotite granite, garnet-cordierite granite and hypersthene granite porphyry. Although there are few systematic geochemical and isotopic studies, the presence of abundant Al-rich minerals, such as cordierite, garnet, hypersthene, almandine, sillimanite, and andalusite, in these rocks suggests that they can be classified as S-type granites of Chappell and White (1974) in the mineralogical sense.

EY magmatism (180-140 Ma) (Zhou and Li, 2000) has resulted in a large number of granitic intrusives forming the Nanling Mountains (NLM). This area, situated in Longitude $110^{\circ} \mathrm{E}-117^{\circ} \mathrm{E}$ and Latitude $22^{\circ} 40^{\prime} \mathrm{N}-26^{\circ} 20^{\prime} \mathrm{N}$, is the watershed of the Yangtze and Pearl Rivers (Fig. 1c). For decades, related mineralization in the NLM granites has been the center of attention because of the existence of some world-class $\mathrm{W}, \mathrm{Si}$, and $\mathrm{Bi}$ ore deposits (Chen and Jahn, 1998; Zhou and Li, 2000; Pirajno and Bagas, 2002; Li et al., 2003, 2004; Xu et al., 2005). Large NLM batholiths are composed predominantly of biotite granite, granodiorite and A-type granite, and sporadically distributed small bodies are mainly gabbro and syenite. They are accompanied by some Late Jurassic bimodal basalts and rhyolites in southern Jiangxi ( $\mathrm{Li}$ et al., 2003). Based on recent geochronological data, ages of the NLM granites appear to be largely concentrated in the range of ca. 165-155 Ma (Li, 2000; Xu et al., 2005).

Products of LY magmatism (140-80 Ma) are mainly restricted in the SE coast of S. China forming a NE-SW magmatic belt about $900 \mathrm{~km}$ long and $150 \mathrm{~km}$ wide (Fig. 1a). Rock types are mainly granodiorite, monzogranite, syenogranite and alkali feldspar granite as a rock complex (110-100 Ma), A-type granites (100-90 Ma) and basalt-rhyolite bimodal volcanics $(90-80 \mathrm{Ma})$ (Chen et al., 2004). Judging from the general appearance of hornblende in the intermediate rocks, as well as the geochemical and $\mathrm{Sr}-\mathrm{Nd}$ isotopic characteristics, LY granitoids are typical I-type granites (Chen et al., 2000). Granites of the Fuzhou and Zhangzhou Complexes (FZC) in Fujian are the most extensively studied representatives of this belt (Fig. 1d).

In this paper, 25 granitic samples and their apatite separates from four batholiths (Pubai, Jiuzhou, Taima, and Nadong) of the DRS granitic suites (Fig. 1b), six batholiths (Jiufeng, Qitianling, Dadongshan, Guidong, Fogang, and Lianyang) of the NLM region (Fig. 1c), and six FZC plutons (Fuzhou, Danyang, and Lianjiang from the Fuzhou Complex and Zudi, Yanqian, and Changtai from the Zhangzhou Complex) of the Fujian Province (Fig. 1d) are studied. Rocks from these places are commonly referred to in the discussion of Mesozoic granitic magmatism of the Cathaysia Block (e.g., Chen and Jahn, 1998; Zhou and Li, 2000; Xu et al., 2005). In addition, samples from one gabbro body (Chebu) and three syenite bodies (Quannan, Paitan, and Ejinao) are also included especially for revealing the apatite geochemistry in magmas with larger involvement of the mantle component ( $\mathrm{Li}$ et al., 2003), in which Paitan and Ejinao bodies are closely associated with the Fogang batholith. Batholith, pluton and igneous body are arbitrarily set by the surface exposure of rocks $>500,500-100$, and $<100 \mathrm{~km}^{2}$.

\section{Analytical methods}

Major element contents of rock samples were determined with an X-ray fluorescence using a Rigaku ${ }^{\circledR}$ RIX 2000 spectrometer on fused glass disk at Department of Geosciences, National Taiwan University (Lee et al., 1997). Trace element abundances were measured by inductively coupled plasma-mass spectrometry (ICP-MS) using a Perkin Elmer ${ }^{\circledR}$ Elan-6000 spectrometer at Guangzhou Institute of Geochemistry, the Chinese Academy of Sciences (Liu et al., 1996). The analytical precision and accuracy are generally better than $5 \%$ for most elements. Sr and $\mathrm{Nd}$ isotope compositions were measured using a Finnigan ${ }^{\circledR}$ MAT 262 mass spectrometer at Department of Earth Sciences, National Cheng Kung University. The isotopic ratios were corrected for mass fractionation by normalizing to ${ }^{86} \mathrm{Sr} /{ }^{88} \mathrm{Sr}=0.1194$ and ${ }^{146} \mathrm{Nd} /{ }^{144} \mathrm{Nd}=0.7219$. Longterm laboratory measurements for SRM $987 \mathrm{Sr}$ and $\mathrm{La}$ Jolla Nd standards yield $0.710239 \pm 0.000012 \quad(n=30)$ and $0.511847 \pm 0.000012(n=30)$, respectively.

Apatite separates were mounted in epoxy and well-polished on the exposed surfaces suitable for both the electron microprobe (EMP) and laser ablation inductively coupled plasma-mass spectrometer (LA-ICP-MS) analyses. Major and minor elements $(\mathrm{Ca}, \mathrm{P}, \mathrm{F}, \mathrm{Cl}, \mathrm{Si}, \mathrm{Na}$, and $\mathrm{S})$ were analyzed using a Shimadzu-ARL EMX-SM7 electron microprobe equipped with four channels of wavelength dispersive spectrometer at National Taiwan University. The analyzing conditions were $15 \mathrm{kV}$ acceleration potential, 
6-8 nA sample current, and a beam spot $\sim 10 \mu \mathrm{m}$ in diameter. Data reduction is based on the ZAF correction procedure (Chen and Tung, 1984). Concentrations of trace elements $(\mathrm{Mn}, \mathrm{Sr}, \mathrm{Ba}, \mathrm{Th}, \mathrm{U}, \mathrm{Pb}$, and $\mathrm{Y})$ including rare earth elements (REE) were determined on single apatites by an excimer LA-ICP-MS at the Key Laboratory of Continental Dynamics, Northwest University, Xi'an, China. The ICP-MS used is an Elan 6100 DRC (Dynamic Reaction Cell) from Perkin Elmer/SCIEX (Canada) coupled to the GeoLas 200M laser-ablation system (MicroLas, Göttingen, Germany) equipped with a $193 \mathrm{~nm}$ ArF-excimer laser and a homogenizing, imaging optical system. Detailed analytical procedures and instrumental operating conditions have been described by Gao et al. (2002). The spot size has been adjusted to $40-60 \mu \mathrm{m}$ in this study. Helium was used as a carrier gas for argon to maintain stable and optimum excitation conditions. Coexisting accessory uraninites, if any, were subjected to EMP analysis for $\mathrm{U}, \mathrm{Th}, \mathrm{Pb}$, and $\mathrm{Y}$.

Calcium content of apatite obtained from EMP analysis was used as an internal standard to correct for differences in the ablation yield between sample and reference materials, matrix effects and signal drift in the ICP-MS. The external standard used for the apatite in situ analysis was NIST SRM 610 (Pearce et al., 1997). Each spot analysis consisted of approximately $30 \mathrm{~s}$ background (gas blank) followed by $60 \mathrm{~s}$ data acquisition from the sample. Trace element concentrations were calculated using GLITTER 4.0 software (Macquarie University). Accuracy and precision of the present LA-ICP-MS setting have been reported for known international standards, including NIST glass standards (SRM 610, 612, and 614) and USGS rock standards (BCR-2G, BHVO-2G and BIR-1G) (Gao et al., 2002). Generally, detection limits for a spot size of $60 \mu \mathrm{m}$ are well below the ppm level for all elements analyzed, and the precision of analyses on the NIST SRM 610 are $1-2.6 \%$ for trace elements in the studied samples.

\section{Whole-rock geochemistry and $\mathrm{Sr}-\mathrm{Nd}$ isotope compositions}

For a large number of Mesozoic intrusive rocks in S. China analyzed, whole-rock geochemical data reported here (Table 1) only include those which have accompanied by apatite data $(n=29)$. Additional major element compositions of the NLM granites $(n=37)$ referred in this study are listed in Appendix. All the $\mathrm{Sr}$ and $\mathrm{Nd}$ isotopic compositions $(n=38)$ are given in Table 2 .

DRS granites are evolved rocks $\left(\mathrm{SiO}_{2}=68-75 \mathrm{wt} \%\right)$, with relatively low $\mathrm{Na}_{2} \mathrm{O}$ contents $(1.6-2.2 \mathrm{wt} \%)$. The aluminum saturation index (ASI, or $\mathrm{A} / \mathrm{CNK}=1.15-1.47$ ) indicates that these rocks are strongly peraluminous (ASI > 1.1, Frost et al., 2001). In contrast, FZC granites have a large variation of silica $\left(\mathrm{SiO}_{2}=58-77 \mathrm{wt} \%\right)$, relatively high $\mathrm{Na}_{2} \mathrm{O}$ contents $(3.0-4.5 \mathrm{wt} \%)$, and are mostly metaluminous (ASI $=0.89-1.04$ ). Voluminous NLM granites also have a wide range of silica $\left(\mathrm{SiO}_{2}=63-78 \mathrm{wt} \%\right)$ or aluminum saturation (ASI $=0.91-1.21)$ but moderate
$\mathrm{Na}_{2} \mathrm{O}$ contents $(2.4-4.0 \mathrm{wt} \%)$, as compared with wider fields of DRS and FZC granites illustrated (Fig. 2a and b). Chebu gabbro $\left(\mathrm{SiO}_{2}=50 \mathrm{wt} \% ; \quad \mathrm{Na}_{2} \mathrm{O}=2.6 \mathrm{wt} \%\right)$ and Quannan syenite $\left(\mathrm{SiO}_{2}=54 \mathrm{wt} \% ; \mathrm{Na}_{2} \mathrm{O}=4.1 \mathrm{wt} \%\right)$ in S. Jiangxi as well as Paitan and Ejino syenites $\left(\mathrm{SiO}_{2}=59-62 \mathrm{wt} \% ; \mathrm{Na}_{2} \mathrm{O}=3.6-7.5 \mathrm{wt} \%\right)$ in Guangdong appear to be Na-rich rocks as a whole. They are grouped together because of a crystal fractionation relationship between gabbro and syenite ( $\mathrm{Li}$ et al., 2003).

The total $\mathrm{FeO}$ and $\mathrm{CaO}$ contents of the studied granites are positively correlated and the DRS, NLM and FZC granites are indistinguishable at lower $\mathrm{CaO}$-total $\mathrm{FeO}$ content. At higher $\mathrm{CaO}$ and total $\mathrm{FeO}$ levels, FZC granites have higher $\mathrm{CaO}$ contents than DRS granites at a given total $\mathrm{FeO}$ content, and NLM granites fall between the FZC and DRS suites (Fig. 2c). Using the $\mathrm{Na}_{2} \mathrm{O}-\mathrm{K}_{2} \mathrm{O}$ divide for differentiating $\mathrm{S}$ - and I-type granites (Chappell and White, 2001), FZC and DRS granites can be assigned to S- and I-types, respectively, whereas NLM granites astride the dividing line (Fig. 2a). Also the ASI (at 1.1) is a very effective parameter to separate FZC from DRS granites, again, NLM granites spread in the middle (Fig. 2b).

Chondrite-normalized REE distribution patterns of all the Mesozoic intrusive rocks in S. China, except gabbro and few syenitic rocks, invariably show gentle LREE enrichment and moderate negative Eu anomalies (Fig. 3). Here we draw attention to the general similarity in the shape and relative abundance of REE distribution patterns for all granitic rocks, probably indicating insignificant feldspar crystal fractionation between the mafic and felsic granites. Only in the gabbro-syenite series, feldspar crystal fractionation is important (Fig. 3c).

$\mathrm{Sr}$ and $\mathrm{Nd}$ isotope compositions of the Mesozoic granitoids in S. China are more variable as compared with Iand S-type granites in LFB (Fig. 4). DRS granites generally exhibit the most enriched character with a small range of $\varepsilon \operatorname{Nd}(T)(-12$ to -14$)$ but a wide range of Isr $(0.722$ 0.730). Such isotopic characteristics, even more enriched than the LFB S-type granites (Fig. 4), have been interpreted as indicating the involvement of sedimentary materials (Shen and Lin, 2002). These rocks also have significantly more enriched $\mathrm{Sr}$ and $\mathrm{Nd}$ isotope compositions than the pre-Mesozoic (predominantly Caledonian) granites that overwhelmed other rocks in the Cathaysia Block before overprinted by the Mesozoic magmatism (see later section). FZC granites, on the other hand, have more depleted $\mathrm{Sr}$ and $\mathrm{Nd}$ isotope compositions with narrower ranges of both $\varepsilon \mathrm{Nd}(T)(-3$ to -6$)$ and Isr (0.7050.708). They conform to I-type granites in the SE China coastal area (Chen et al., 2000), and overlap with the more enriched field of I-type granites in LFB (Collins, 1996).

In the NLM region, gabbro, and syenites have $\mathrm{Sr}$ and $\mathrm{Nd}$ isotope compositions $(\varepsilon \mathrm{Nd}(T)=3.0$ to -2.6 and Isr $=0.704-0.708)$ consistent with similar rocks reported by Li et al. (2003). They overlap with more depleted LFB I-type granites, but can be clearly distinguished from 
Table 1

Chemical compositions of representative Darongshan (DRS), Fuzhou-Zhangzhou Complex (FZC), Nanling Mountains (NLM) granites, gabbro and syenites of S. China

\begin{tabular}{|c|c|c|c|c|c|c|c|c|c|c|c|c|c|}
\hline \multirow{5}{*}{$\begin{array}{l}\text { Locality: } \\
\text { Sample no.: } \\
\text { Latitude }\left({ }^{\circ} \mathrm{N}\right): \\
\text { Longitude }\left({ }^{\circ} \mathrm{E}\right) \text { : }\end{array}$} & \multicolumn{7}{|c|}{ DRS granites } & \multicolumn{6}{|c|}{ FZC granites } \\
\hline & Jiuzhou & Taima & Pubai & Pubai & Nadong & Nadong & Nadong & Zudi & Changtai & Danyang & Fuzhou & Yanqian & Lianjiang \\
\hline & $22 \mathrm{GX} 12$ & $22 \mathrm{GX} 01$ & $22 \mathrm{GX} 13$ & 22GX16 & $22 \mathrm{GX} 07$ & $22 \mathrm{GX} 03$ & $22 \mathrm{GX} 04$ & 93ZUD01 & $93 \mathrm{CHT} 01$ & $92 \mathrm{BD} 01$ & 92KS01 & 93YAN01 & 92GT01 \\
\hline & $22^{\circ} 08^{\prime}$ & $22^{\circ} 08^{\prime}$ & $22^{\circ} 20^{\prime}$ & $22^{\circ} 24^{\prime}$ & $21^{\circ} 42^{\prime}$ & $21^{\circ} 43^{\prime}$ & $21^{\circ} 41^{\prime}$ & $24^{\circ} 40^{\prime}$ & $24^{\circ} 38^{\prime}$ & $26^{\circ} 28^{\prime}$ & $26^{\circ} 05^{\prime}$ & $24^{\circ} 44^{\prime}$ & $26^{\circ} 11^{\prime}$ \\
\hline & $108^{\circ} 35^{\prime}$ & $108^{\circ} 26^{\prime}$ & $109^{\circ} 18^{\prime}$ & $109^{\circ} 34^{\prime}$ & $107^{\circ} 47^{\prime}$ & $108^{\circ} 03^{\prime}$ & $107^{\circ} 58^{\prime}$ & $117^{\circ} 49^{\prime}$ & $117^{\circ} 46^{\prime}$ & $119^{\circ} 29^{\prime}$ & $119^{\circ} 24^{\prime}$ & $117^{\circ} 47^{\prime}$ & $119^{\circ} 33^{\prime}$ \\
\hline \multicolumn{14}{|l|}{$w t \%$} \\
\hline $\mathrm{SiO}_{2}$ & 68.05 & 70.68 & 70.40 & 71.24 & 71.25 & 73.67 & 74.47 & 58.61 & 63.26 & 67.54 & 74.94 & 75.57 & 77.04 \\
\hline $\mathrm{TiO}_{2}$ & 0.73 & 0.42 & 0.50 & 0.42 & 0.52 & 0.30 & 0.32 & 0.90 & 0.69 & 0.52 & 0.21 & 0.16 & 0.13 \\
\hline $\mathrm{Al}_{2} \mathrm{O}_{3}$ & 14.34 & 14.72 & 14.61 & 14.27 & 13.98 & 13.22 & 12.96 & 17.40 & 17.27 & 15.89 & 13.96 & 13.22 & 13.07 \\
\hline $\mathrm{Fe}_{2} \mathrm{O}_{3}$ & 4.79 & 2.50 & 3.43 & 3.32 & 3.41 & 1.77 & 1.90 & 7.45 & 5.08 & 3.03 & 1.17 & 1.04 & 0.78 \\
\hline $\mathrm{MnO}$ & 0.05 & 0.03 & 0.04 & 0.03 & 0.04 & 0.02 & 0.03 & 0.12 & 0.08 & 0.06 & 0.07 & 0.05 & 0.07 \\
\hline $\mathrm{MgO}$ & 1.57 & 0.49 & 1.01 & 1.02 & 0.67 & 0.29 & 0.30 & 3.49 & 1.77 & 0.95 & 0.16 & 0.09 & 0.00 \\
\hline $\mathrm{CaO}$ & 2.02 & 2.15 & 1.47 & 1.26 & 1.73 & 1.34 & 1.23 & 6.49 & 4.75 & 2.81 & 1.08 & 0.96 & 0.50 \\
\hline $\mathrm{Na}_{2} \mathrm{O}$ & 1.61 & 1.89 & 1.73 & 1.77 & 2.05 & 2.16 & 2.06 & 2.99 & 3.39 & 3.69 & 4.18 & 3.57 & 4.46 \\
\hline $\mathrm{K}_{2} \mathrm{O}$ & 3.88 & 4.91 & 4.26 & 4.14 & 4.80 & 5.05 & 4.96 & 2.65 & 2.84 & 4.68 & 4.24 & 4.92 & 4.38 \\
\hline $\mathrm{P}_{2} \mathrm{O}_{5}$ & 0.15 & 0.14 & 0.20 & 0.15 & 0.13 & 0.08 & 0.08 & 0.25 & 0.23 & 0.15 & 0.07 & 0.06 & 0.04 \\
\hline Total & 97.19 & 97.93 & 97.65 & 97.62 & 98.58 & 97.90 & 98.31 & 100.35 & 99.36 & 99.32 & 100.08 & 99.64 & 100.47 \\
\hline ASI & 1.36 & 1.19 & 1.44 & 1.47 & 1.19 & 1.15 & 1.18 & 0.89 & 1.00 & 0.98 & 1.04 & 1.02 & 1.01 \\
\hline $\mathrm{A} / \mathrm{NK}$ & 2.09 & 1.75 & 1.96 & 1.93 & 1.63 & 1.47 & 1.48 & 2.23 & 2.00 & 1.43 & 1.22 & 1.18 & 1.08 \\
\hline \multicolumn{14}{|l|}{ ppm } \\
\hline $\mathrm{Ni}$ & 21.2 & 6.14 & 11.3 & 12.3 & 8.10 & 4.58 & 4.82 & 16.2 & 2.74 & 3.65 & n.d. & n.d. & n.d. \\
\hline $\mathrm{Ga}$ & 17.9 & 18.0 & 17.5 & 17.0 & 17.5 & 17.1 & 16.8 & 18.0 & 19.0 & 16.9 & 13.5 & 14.3 & 16.8 \\
\hline $\mathrm{Rb}$ & 195 & 242 & 236 & 241 & 266 & 322 & 337 & 134 & 134 & 185 & 145 & 266 & 157 \\
\hline $\mathrm{Sr}$ & 109 & 95.4 & 78.0 & 70.8 & 78.8 & 45.6 & 38.9 & 512 & 599 & 408 & 159 & 75.5 & 33.4 \\
\hline $\mathrm{Y}$ & 35.8 & 42.7 & 43.2 & 37.7 & 44.6 & 44.2 & 103 & 26.5 & 19.0 & 26.9 & 18.1 & 15.2 & 28.8 \\
\hline $\mathrm{Zr}$ & 274 & 220 & 219 & 194 & 279 & 171 & 186 & 222 & 175 & 251 & 117 & 108 & 119 \\
\hline $\mathrm{Nb}$ & 15.7 & 12.0 & 14.9 & 13.3 & 14.2 & 10.7 & 11.2 & 10.3 & 9.28 & 13.3 & 11.3 & 14.5 & 19.6 \\
\hline Cs & 10.0 & 10.2 & 19.6 & 14.7 & 9.23 & 21.1 & 18.5 & 9.08 & 7.17 & 8.39 & 1.51 & 6.09 & 1.20 \\
\hline $\mathrm{Ba}$ & 631 & 758 & 498 & 396 & 661 & 375 & 291 & 782 & 806 & 1155 & 1409 & 167 & 339 \\
\hline $\mathrm{La}$ & 46.7 & 46.7 & 43.0 & 30.2 & 50.8 & 42.0 & 43.3 & 28.3 & 34.0 & 45.9 & 30.4 & 30.4 & 26.9 \\
\hline $\mathrm{Ce}$ & 93.4 & 92.0 & 88.1 & 59.2 & 101 & 83.2 & 84.1 & 60.0 & 67.0 & 88.5 & 57.4 & 47.2 & 52.5 \\
\hline $\operatorname{Pr}$ & 10.6 & 11.1 & 10.2 & 7.13 & 11.3 & 9.87 & 10.5 & 7.59 & 8.08 & 10.3 & 6.33 & 4.71 & 5.85 \\
\hline $\mathrm{Nd}$ & 39.6 & 41.3 & 38.2 & 26.3 & 42.0 & 36.0 & 39.0 & 28.9 & 29.6 & 35.8 & 20.7 & 14.1 & 18.6 \\
\hline $\mathrm{Sm}$ & 7.80 & 8.53 & 8.01 & 5.85 & 8.50 & 7.48 & 9.43 & 5.89 & 5.52 & 6.39 & 3.57 & 2.23 & 3.41 \\
\hline $\mathrm{Eu}$ & 1.26 & 1.31 & 1.00 & 0.865 & 1.16 & 0.794 & 1.05 & 1.27 & 1.18 & 1.16 & 0.800 & 0.377 & 0.430 \\
\hline $\mathrm{Gd}$ & 7.50 & 8.14 & 7.71 & 5.92 & 8.38 & 7.40 & 11.7 & 5.19 & 4.53 & 5.27 & 3.05 & 2.18 & 3.15 \\
\hline $\mathrm{Tb}$ & 1.12 & 1.30 & 1.27 & 1.04 & 1.32 & 1.23 & 2.40 & 0.804 & 0.642 & 0.784 & 0.460 & 0.312 & 0.550 \\
\hline Dy & 6.11 & 7.05 & 7.13 & 6.12 & 7.34 & 6.93 & 14.9 & 4.45 & 3.28 & 4.31 & 2.63 & 1.84 & 3.51 \\
\hline Но & 1.18 & 1.49 & 1.51 & 1.28 & 1.50 & 1.51 & 3.62 & 0.880 & 0.611 & 0.864 & 0.548 & 0.41 & 0.796 \\
\hline $\mathrm{Er}$ & 3.25 & 4.09 & 4.14 & 3.46 & 4.09 & 4.11 & 9.67 & 2.39 & 1.66 & 2.45 & 1.64 & 1.32 & 2.51 \\
\hline $\mathrm{Tm}$ & 0.509 & 0.601 & 0.635 & 0.518 & 0.619 & 0.640 & 1.46 & 0.352 & 0.235 & 0.366 & 0.257 & 0.232 & 0.406 \\
\hline $\mathrm{Yb}$ & 3.40 & 3.88 & 4.03 & 3.21 & 3.97 & 4.02 & 8.37 & 2.20 & 1.47 & 2.43 & 1.81 & 1.72 & 2.80 \\
\hline $\mathrm{Lu}$ & 0.579 & 0.604 & 0.644 & 0.504 & 0.646 & 0.633 & 1.29 & 0.338 & 0.222 & 0.384 & 0.290 & 0.300 & 0.451 \\
\hline $\mathrm{Hf}$ & 6.80 & 6.17 & 5.77 & 5.38 & 6.83 & 4.94 & 5.28 & 5.72 & 4.86 & 6.45 & 3.49 & 3.83 & 4.42 \\
\hline Та & 1.22 & 1.13 & 1.28 & 1.21 & 1.17 & 1.10 & 1.14 & 0.841 & 0.817 & 1.01 & 0.914 & 1.01 & 1.32 \\
\hline $\mathrm{Pb}$ & 29.1 & 36.4 & 28.8 & 29.4 & 33.1 & 36.7 & 34.9 & 19.3 & 17.5 & 28.1 & 21.4 & 39.4 & 28.3 \\
\hline Th & 22.1 & 25.5 & 21.2 & 16.0 & 26.4 & 28.3 & 29.8 & 23.1 & 26.8 & 21.4 & 17.2 & 38.0 & 13.3 \\
\hline \multirow[t]{2}{*}{$\mathrm{U}$} & 5.54 & 6.35 & 4.40 & 4.25 & 6.38 & 6.76 & 7.53 & 5.82 & 6.46 & 3.98 & 4.22 & 11.8 & 3.64 \\
\hline & & & & & & & & & & & & \multicolumn{2}{|c|}{ (continued on next page) } \\
\hline
\end{tabular}


Table 1 (continued)

\begin{tabular}{|c|c|c|c|c|c|c|c|c|c|c|c|c|}
\hline \multirow{5}{*}{$\begin{array}{l}\text { Locality: } \\
\text { Sample no.: } \\
\text { Latitude }\left({ }^{\circ} \mathrm{N}\right) \text { : } \\
\text { Longitude }\left({ }^{\circ} \mathrm{E}\right) \text { : }\end{array}$} & \multicolumn{12}{|c|}{ NLM granites } \\
\hline & Guidong & Guidong & Guidong & Fogang & Fogang & Fogang & Fogang & Qitianling & Lianyang & Jiufeng & Jiufeng & Dadongshan \\
\hline & 99GD16 & 99GD17 & $99 \mathrm{GD} 18 \mathrm{~b}$ & 99GD36a & 99GD02a & 99GD03b & 99GD19 & 21HUN01 & $21 \mathrm{GD} 01 \mathrm{a}$ & 97GD61 & 97GD54 & 21GD07 \\
\hline & $24^{\circ} 32^{\prime}$ & $24^{\circ} 32^{\prime}$ & $24^{\circ} 42^{\prime}$ & $24^{\circ} 01^{\prime}$ & $23^{\circ} 16^{\prime}$ & $23^{\circ} 56^{\prime}$ & $24^{\circ} 08^{\prime}$ & $25^{\circ} 33^{\prime}$ & $24^{\circ} 19^{\prime}$ & $25^{\circ} 16^{\prime}$ & $25^{\circ} 20^{\prime}$ & $24^{\circ} 45^{\prime}$ \\
\hline & $114^{\circ} 11^{\prime}$ & $114^{\circ} 11^{\prime}$ & $114^{\circ} 04^{\prime}$ & $114^{\circ} 04^{\prime}$ & $113^{\circ} 45^{\prime}$ & $114^{\circ} 48^{\prime}$ & $113^{\circ} 55^{\prime}$ & $112^{\circ} 59^{\prime}$ & $112^{\circ} 07^{\prime}$ & $113^{\circ} 29^{\prime}$ & $113^{\circ} 18^{\prime}$ & $112^{\circ} 51^{\prime}$ \\
\hline \multicolumn{13}{|l|}{$w t \%$} \\
\hline $\mathrm{SiO}_{2}$ & 63.34 & 64.77 & 73.53 & 66.11 & 70.89 & 71.02 & 75.56 & 66.18 & 66.67 & 69.03 & 71.66 & 77.43 \\
\hline $\mathrm{TiO}_{2}$ & 0.71 & 0.58 & 0.15 & 0.59 & 0.43 & 0.29 & 0.15 & 0.58 & 0.36 & 0.52 & 0.31 & 0.11 \\
\hline $\mathrm{Al}_{2} \mathrm{O}_{3}$ & 16.63 & 16.83 & 14.64 & 15.75 & 14.75 & 14.60 & 12.57 & 14.43 & 15.82 & 15.16 & 14.63 & 11.18 \\
\hline $\mathrm{Fe}_{2} \mathrm{O}_{3}$ & 4.66 & 3.94 & 1.19 & 4.14 & 2.63 & 2.37 & 1.53 & 4.20 & 2.55 & 3.50 & 2.19 & 0.78 \\
\hline $\mathrm{MnO}$ & 0.08 & 0.07 & 0.05 & 0.06 & 0.05 & 0.05 & 0.05 & 0.06 & 0.05 & 0.08 & 0.06 & 0.02 \\
\hline $\mathrm{MgO}$ & 1.79 & 1.43 & 0.02 & 0.75 & 0.33 & 0.22 & b.d.l. & 0.58 & 1.07 & 1.06 & 0.54 & 0.09 \\
\hline $\mathrm{CaO}$ & 3.68 & 3.26 & 0.93 & 2.47 & 1.54 & 1.89 & 1.03 & 2.22 & 2.34 & 2.76 & 1.81 & 0.71 \\
\hline $\mathrm{Na}_{2} \mathrm{O}$ & 3.09 & 3.03 & 2.97 & 2.59 & 2.42 & 2.92 & 2.82 & 3.57 & 4.00 & 2.86 & 3.00 & 2.95 \\
\hline $\mathrm{K}_{2} \mathrm{O}$ & 3.82 & 4.46 & 5.10 & 5.64 & 5.53 & 5.02 & 4.71 & 5.43 & 5.05 & 4.37 & 5.34 & 4.88 \\
\hline $\mathrm{P}_{2} \mathrm{O}_{5}$ & 0.28 & 0.24 & 0.15 & 0.17 & 0.23 & 0.09 & 0.05 & 0.20 & 0.13 & 0.17 & 0.13 & 0.05 \\
\hline Total & 98.08 & 98.61 & 98.73 & 98.27 & 98.80 & 98.47 & 98.47 & 97.45 & 98.04 & 99.51 & 99.67 & 98.20 \\
\hline ASI & 1.04 & 1.07 & 1.21 & 1.06 & 1.15 & 1.07 & 1.08 & 0.91 & 0.97 & 1.05 & 1.04 & 0.98 \\
\hline $\mathrm{A} / \mathrm{NK}$ & 1.87 & 1.72 & 1.47 & 1.52 & 1.48 & 1.43 & 1.29 & 1.23 & 1.31 & 1.61 & 1.37 & 1.10 \\
\hline \multicolumn{13}{|l|}{ ppm } \\
\hline $\mathrm{Ni}$ & 9.67 & 7.28 & 0.329 & 0.218 & 0.394 & 0.767 & 0.532 & 3.30 & 12.8 & 6.62 & 7.46 & 1.65 \\
\hline $\mathrm{Ga}$ & 22.2 & 22.2 & 19.0 & 22.1 & 8.59 & 27.1 & 17.9 & 21.1 & 15.0 & 17.9 & 20.9 & 12.5 \\
\hline $\mathrm{Rb}$ & 216 & 218 & 396 & 257 & 139 & 429 & 279 & 175 & 310 & 267 & 448 & 356 \\
\hline $\mathrm{Sr}$ & 324 & 345 & 52.4 & 152 & 58.9 & 182 & 65.5 & 48.9 & 136 & 221 & 148 & 26.8 \\
\hline Y & 26.7 & 22.2 & 27.2 & 50.0 & 14.2 & 51.1 & 43.8 & 279 & 17.2 & 30.5 & 30.4 & 42.7 \\
\hline $\mathrm{Zr}$ & 250 & 216 & 86.7 & 247 & 96.3 & 234 & 156 & 30.7 & 214 & 175 & 163 & 96.6 \\
\hline $\mathrm{Nb}$ & 16.2 & 13.1 & 23.4 & 32.1 & 8.53 & 28.5 & 25.4 & b.d.l. & 13.8 & 20.5 & 26.8 & 16.7 \\
\hline Cs & 12.8 & 11.3 & 32.3 & 8.69 & 2.65 & 17.6 & 3.39 & 28.0 & 24.1 & 18.7 & 56.9 & 18.4 \\
\hline $\mathrm{Ba}$ & 943 & 1219 & 266 & 833 & 261 & 609 & 183 & 792 & 688 & 686 & 669 & 72.0 \\
\hline $\mathrm{La}$ & 66.3 & 75.9 & 23.9 & 73.0 & 22.1 & 60.1 & 47.6 & 62.0 & 20.2 & 31.1 & 47.7 & 34.4 \\
\hline $\mathrm{Ce}$ & 127 & 142 & 51.7 & 142 & 49.3 & 119 & 100 & 124 & 39.0 & 64.4 & 89.4 & 52.80 \\
\hline $\operatorname{Pr}$ & 14.7 & 15.9 & 6.27 & 16.4 & 6.06 & 13.5 & 12.0 & 15.0 & 4.70 & 8.03 & 9.98 & 8.87 \\
\hline $\mathrm{Nd}$ & 54.6 & 55.3 & 20.8 & 57.6 & 21.4 & 47.5 & 41.8 & 55.8 & 17.1 & 29.0 & 32.9 & 32.2 \\
\hline $\mathrm{Sm}$ & 9.61 & 8.77 & 4.88 & 11.8 & 4.23 & 10.0 & 9.21 & 11.0 & 3.31 & 6.08 & 6.25 & 7.76 \\
\hline $\mathrm{Eu}$ & 1.59 & 1.61 & 0.437 & 1.01 & 0.434 & 1.09 & 0.448 & 1.75 & 0.860 & 1.10 & 0.900 & 0.370 \\
\hline $\mathrm{Gd}$ & 6.56 & 5.82 & 5.09 & 10.1 & 3.85 & 8.33 & 8.21 & 10.0 & 3.11 & 5.20 & 5.60 & 6.79 \\
\hline $\mathrm{Tb}$ & 0.971 & 0.836 & 0.897 & 1.67 & 0.577 & 1.44 & 1.41 & 1.47 & 0.460 & 0.850 & 0.847 & 1.17 \\
\hline Dy & 5.20 & 4.52 & 4.95 & 9.79 & 2.92 & 8.46 & 8.02 & 8.00 & 2.62 & 5.19 & 5.02 & 6.80 \\
\hline Но & 1.00 & 0.838 & 0.950 & 1.78 & 0.542 & 1.73 & 1.57 & 1.63 & 0.560 & 1.03 & 0.972 & 1.42 \\
\hline $\mathrm{Er}$ & 2.71 & 2.22 & 2.65 & 4.87 & 1.37 & 5.06 & 4.67 & 4.53 & 1.66 & 2.87 & 2.81 & 4.09 \\
\hline $\mathrm{Tm}$ & 0.383 & 0.315 & 0.403 & 0.675 & 0.207 & 0.814 & 0.704 & 0.680 & 0.270 & 0.477 & 0.472 & 0.670 \\
\hline $\mathrm{Yb}$ & 2.35 & 1.99 & 2.50 & 3.88 & 1.21 & 5.33 & 4.36 & 4.43 & 1.97 & 2.96 & 2.96 & 4.37 \\
\hline $\mathrm{Lu}$ & 0.361 & 0.296 & 0.360 & 0.569 & 0.179 & 0.840 & 0.668 & 0.730 & 0.350 & 0.437 & 0.466 & 0.710 \\
\hline $\mathrm{Hf}$ & 7.25 & 6.10 & 3.06 & 7.85 & 2.72 & 7.97 & 5.76 & 7.61 & 6.30 & 5.01 & 5.20 & 4.06 \\
\hline $\mathrm{Ta}$ & 1.44 & 1.14 & 5.34 & 3.27 & 0.846 & 3.74 & 2.68 & 2.64 & 1.65 & 3.03 & 4.95 & 2.82 \\
\hline $\mathrm{Pb}$ & 39.2 & 46.4 & 39.2 & 24.0 & 15.5 & 44.0 & 28.7 & 37.9 & 66.3 & n.d. & n.d. & 44.3 \\
\hline Th & 24.7 & 27.5 & 26.5 & 38.1 & 17.0 & 77.1 & 66.2 & 30.0 & 32.9 & 26.2 & 45.4 & 45.1 \\
\hline $\mathrm{U}$ & 4.60 & 3.86 & 27.5 & 14.9 & 3.43 & 21.3 & 18.7 & 7.35 & 9.04 & 10.0 & 21.8 & 10.7 \\
\hline
\end{tabular}


NLM gabbro and syenites

\begin{tabular}{|c|c|c|c|c|}
\hline & & & & \\
\hline Locality: & Chebu & Quannan & Paitan & Ejinao \\
\hline Sample no.: & 99CB02 & 99QN06 & 97GD77 & 97GD84 \\
\hline Latitude $\left({ }^{\circ} \mathrm{N}\right)$ : & $24^{\circ} 50^{\prime}$ & $24^{\circ} 46^{\prime}$ & $23^{\circ} 30^{\prime}$ & $23^{\circ} 43^{\prime}$ \\
\hline Longitude $\left({ }^{\circ} \mathrm{E}\right)$ : & $115^{\circ} 02^{\prime}$ & $114^{\circ} 29^{\prime}$ & $113^{\circ} 47^{\prime}$ & $113^{\circ} 38^{\prime}$ \\
\hline \multicolumn{5}{|l|}{$w t \%$} \\
\hline $\mathrm{SiO}_{2}$ & 49.87 & 54.25 & 62.14 & 59.18 \\
\hline $\mathrm{TiO}_{2}$ & 1.91 & 2.06 & 0.71 & 0.24 \\
\hline $\mathrm{Al}_{2} \mathrm{O}_{3}$ & 15.79 & 16.88 & 15.92 & 18.94 \\
\hline $\mathrm{Fe}_{2} \mathrm{O}_{3}$ & 11.69 & 9.52 & 5.61 & 5.14 \\
\hline $\mathrm{MnO}$ & 0.16 & 0.19 & 0.12 & 0.23 \\
\hline $\mathrm{MgO}$ & 6.54 & 2.57 & 0.73 & 0.36 \\
\hline $\mathrm{CaO}$ & 9.44 & 6.31 & 2.90 & 1.70 \\
\hline $\mathrm{Na}_{2} \mathrm{O}$ & 2.55 & 4.11 & 3.58 & 7.45 \\
\hline $\mathrm{K}_{2} \mathrm{O}$ & 1.11 & 2.83 & 5.75 & 5.62 \\
\hline $\mathrm{P}_{2} \mathrm{O}_{5}$ & 0.28 & 0.65 & 0.25 & 0.10 \\
\hline Total & 100.29 & 99.77 & 98.30 & 100.05 \\
\hline ASI & 0.70 & 0.79 & 0.92 & 0.88 \\
\hline $\mathrm{A} / \mathrm{NK}$ & 2.93 & 1.72 & 1.31 & 1.03 \\
\hline \multicolumn{5}{|l|}{ ppm } \\
\hline $\mathrm{Ni}$ & n.d. & 0.882 & 37.2 & 3.67 \\
\hline $\mathrm{Ga}$ & n.d. & 22.8 & 22.5 & 23.2 \\
\hline $\mathrm{Rb}$ & 58.8 & 94.9 & 135 & 264 \\
\hline $\mathrm{Sr}$ & 307 & 615 & 236 & 66.2 \\
\hline $\mathrm{Y}$ & 32.2 & 35.5 & 32.7 & 26.2 \\
\hline $\mathrm{Zr}$ & 104 & 245 & 578 & 339 \\
\hline $\mathrm{Nb}$ & 19.7 & 59.3 & 37.0 & 69.3 \\
\hline Cs & 1.69 & 6.78 & 3.17 & 3.08 \\
\hline $\mathrm{Ba}$ & 202 & 790 & 997 & 132 \\
\hline $\mathrm{La}$ & 18.9 & 51.6 & 63.2 & 90.2 \\
\hline $\mathrm{Ce}$ & 40.3 & 101 & 124 & 156 \\
\hline $\operatorname{Pr}$ & 5.29 & 12.4 & 14.8 & 16.2 \\
\hline $\mathrm{Nd}$ & 22.2 & 49.1 & 54.8 & 50.0 \\
\hline Sm & 5.09 & 10.0 & 10.3 & 7.01 \\
\hline $\mathrm{Eu}$ & 1.48 & 2.94 & 2.49 & 0.533 \\
\hline $\mathrm{Gd}$ & 5.29 & 8.76 & 7.89 & 6.02 \\
\hline $\mathrm{Tb}$ & 0.850 & 1.33 & 1.23 & 0.835 \\
\hline Dy & 4.99 & 7.39 & 6.52 & 4.63 \\
\hline Ho & 1.05 & 1.43 & 1.25 & 0.914 \\
\hline $\mathrm{Er}$ & 2.99 & 3.87 & 3.51 & 2.77 \\
\hline $\mathrm{Tm}$ & 0.413 & 0.544 & 0.510 & 0.456 \\
\hline $\mathrm{Yb}$ & 2.54 & 3.40 & 3.24 & 3.08 \\
\hline $\mathrm{Lu}$ & 0.378 & 0.484 & 0.497 & 0.518 \\
\hline $\mathrm{Hf}$ & 2.60 & 6.97 & 13.2 & 8.12 \\
\hline $\mathrm{Ta}$ & 1.17 & 4.09 & 2.07 & 2.75 \\
\hline $\mathrm{Pb}$ & 4.91 & 23.0 & 15.8 & n.d. \\
\hline Th & 5.51 & 11.2 & 15.6 & 17.1 \\
\hline $\mathrm{U}$ & 1.27 & 3.50 & 3.25 & 3.17 \\
\hline
\end{tabular}

$\mathrm{ASI}=\left\{\operatorname{molar}\left[\mathrm{Al}_{2} \mathrm{O}_{3} /\left(\mathrm{CaO}+\mathrm{Na}_{2} \mathrm{O}+\mathrm{K}_{2} \mathrm{O}\right)\right]\right\}$ and $\mathrm{A} / \mathrm{NK}=\left\{\operatorname{molar}\left[\mathrm{Al}_{2} \mathrm{O}_{3} /\left(\mathrm{Na}_{2} \mathrm{O}+\mathrm{K}_{2} \mathrm{O}\right)\right]\right\}$.

Major element results of FZC granites are taken from the work of Chen et al., 2000.

n.d., not determined.

b.d.1., below detection limit. 
Table 2

$\mathrm{Sr}$ and Nd isotope compositions of Darongshan (DRS), Fuzhou-Zhangzhou Complex (FZC), Nanling Mountains (NLM) granites, gabbro and syenites of S. China

\begin{tabular}{|c|c|c|c|c|c|c|c|c|c|c|c|c|c|c|}
\hline Locality & Sample & $\mathrm{SiO}_{2}(\mathrm{wt} \%)$ & $\mathrm{Rb}(\mathrm{ppm})$ & $\mathrm{Sr}(\mathrm{ppm})$ & $\mathrm{Sm}(\mathrm{ppm})$ & $\mathrm{Nd}(\mathrm{ppm})$ & ${ }^{87} \mathrm{Rb} /{ }^{86} \mathrm{Sr}$ & ${ }^{87} \mathrm{Sr} /{ }^{86} \mathrm{Sr}$ & $2 \sigma$ & ${ }^{147} \mathrm{Sm} /{ }^{144} \mathrm{Nd}$ & ${ }^{143} \mathrm{Nd} /{ }^{144} \mathrm{Nd}$ & $2 \sigma$ & Isr & $\varepsilon \mathrm{Nd}(T)$ \\
\hline \multicolumn{15}{|c|}{ DRS granites $(T=230 \mathrm{Ma})$} \\
\hline Jiuzhou & $22 \mathrm{GX} 12$ & 68.05 & 195 & 109 & 7.80 & 39.6 & 5.19 & 0.742478 & 10 & 0.1191 & 0.511852 & 12 & 0.725500 & -13.1 \\
\hline Taima & $22 \mathrm{GX} 01$ & 70.68 & 242 & 95.4 & 8.53 & 41.3 & 7.35 & 0.747977 & 10 & 0.1249 & 0.511862 & 12 & 0.723948 & -13.1 \\
\hline Pubai & $22 \mathrm{GX} 13$ & 70.40 & 236 & 78.0 & 8.01 & 38.2 & 8.80 & 0.757267 & 14 & 0.1267 & 0.511812 & 12 & 0.728479 & -14.1 \\
\hline Nadong & $22 \mathrm{GX} 07$ & 71.25 & 266 & 78.8 & 8.50 & 42.0 & 9.81 & 0.754211 & 8 & 0.1224 & 0.511880 & 12 & 0.722119 & -12.7 \\
\hline \multicolumn{15}{|c|}{ FZC granites $(T=110 \mathrm{Ma})$} \\
\hline Zudi & 93ZUD01 & 58.61 & 134 & 512 & 5.89 & 28.9 & 0.758 & 0.707164 & 12 & 0.1232 & 0.512423 & 12 & 0.705979 & -3.2 \\
\hline Changtai & 93СНТ01 & 63.26 & 134 & 599 & 5.52 & 29.6 & 0.648 & 0.706770 & 11 & 0.1127 & 0.512410 & 12 & 0.705757 & -3.3 \\
\hline Danyang & 92BD01 & 67.54 & 185 & 408 & 6.39 & 35.8 & 1.31 & 0.709549 & 11 & 0.1079 & 0.512281 & 12 & 0.707497 & -5.7 \\
\hline Fuzhou & 92KS01 & 74.94 & 145 & 159 & 3.57 & 20.7 & 2.64 & 0.711478 & 10 & 0.1043 & 0.512269 & 12 & 0.707350 & -5.9 \\
\hline Yanqian & 93YAN01 & 75.57 & 266 & 75.5 & 2.23 & 14.1 & 10.2 & 0.720960 & 12 & 0.0956 & 0.512340 & 11 & 0.705012 & -4.4 \\
\hline Lianjiang & 92GT01 & 77.04 & 157 & 33.4 & 3.41 & 18.6 & 13.6 & N.A. & & 0.1108 & 0.512358 & 11 & & -4.3 \\
\hline \multicolumn{15}{|c|}{$N L M$ granites $(T=160 \mathrm{Ma})$} \\
\hline \multirow[t]{4}{*}{ Guidong } & 99GD14 & 63.46 & 197 & 301 & 9.00 & 48.9 & 1.90 & 0.726641 & 11 & 0.1114 & 0.511950 & 12 & 0.722330 & -11.7 \\
\hline & 99GD16 & 63.34 & 216 & 324 & 9.61 & 54.6 & 1.93 & 0.726703 & 13 & 0.1064 & 0.511958 & 10 & 0.722303 & -11.5 \\
\hline & 99GD17 & 64.77 & 218 & 345 & 8.77 & 55.3 & 1.83 & 0.726501 & 12 & 0.0959 & 0.511936 & 10 & 0.722339 & -11.7 \\
\hline & 99GD18b & 73.53 & 396 & 52.4 & 4.88 & 20.8 & 17.5 & N.A. & & 0.1418 & 0.511947 & 13 & & -12.4 \\
\hline \multirow[t]{10}{*}{ Fogang } & 99GD36a & 66.11 & 257 & 152 & 11.8 & 57.6 & 4.90 & 0.726432 & 11 & 0.1239 & 0.512005 & 11 & 0.715287 & -10.9 \\
\hline & 97GD93 & 69.81 & 304 & 89.4 & 10.5 & 60.6 & 9.84 & 0.736744 & 14 & 0.1043 & 0.512045 & 12 & 0.714358 & -9.7 \\
\hline & 99GD04a & 70.10 & 259 & 731 & 8.92 & 49.3 & 1.03 & 0.718974 & 12 & 0.1093 & 0.511963 & 12 & 0.716640 & -11.4 \\
\hline & 97GD51 & 70.80 & 202 & 171 & 6.57 & 40.0 & 3.42 & 0.719924 & 13 & 0.0993 & 0.512040 & 13 & 0.712144 & -9.7 \\
\hline & 99GD02a & 70.89 & 139 & 58.9 & 4.23 & 21.4 & 6.86 & 0.731616 & 16 & 0.1196 & 0.511965 & 10 & 0.716023 & -11.6 \\
\hline & 99GD03b & 71.02 & 429 & 182 & 10.0 & 47.5 & 6.82 & 0.729667 & 14 & 0.1276 & 0.512105 & 10 & 0.714147 & -9.0 \\
\hline & 97GD79 & 74.92 & 226 & 72.1 & 4.72 & 21.2 & 9.09 & 0.741271 & 14 & 0.1346 & 0.511987 & 11 & 0.720589 & -11.5 \\
\hline & 99GD22 & 75.14 & 277 & 74.4 & 7.74 & 38.2 & 10.8 & 0.739791 & 14 & 0.1224 & 0.512033 & 10 & 0.715288 & -10.3 \\
\hline & 99GD19 & 75.56 & 279 & 65.5 & 9.21 & 41.8 & 12.3 & 0.743255 & 16 & 0.1331 & 0.512124 & 11 & 0.715216 & -8.8 \\
\hline & 97GD82 & 77.57 & 256 & 21.1 & 11.2 & 44.3 & 35.1 & N.A. & & 0.1526 & 0.512128 & 11 & & -9.1 \\
\hline \multirow[t]{2}{*}{ Qitianling } & 21HUN01 & 66.18 & 175 & 48.9 & 11.0 & 55.8 & 10.4 & N.A. & & 0.1192 & 0.512220 & 12 & & -6.6 \\
\hline & 21HUN02 & 68.01 & 189 & 41.1 & 8.28 & 37.9 & 13.3 & N.A. & & 0.1321 & 0.512182 & 12 & & -7.6 \\
\hline \multirow[t]{2}{*}{ Lianyang } & 21GD01a & 66.67 & 310 & 136 & 3.31 & 17.1 & 6.60 & N.A. & & 0.1170 & 0.512060 & 11 & & -9.7 \\
\hline & $21 \mathrm{GD} 02 \mathrm{~b}$ & 74.83 & 276 & 34.4 & 3.28 & 12.8 & 23.2 & N.A. & & 0.1549 & 0.512104 & 12 & & -9.6 \\
\hline \multirow[t]{3}{*}{ Jiufeng } & 97GD53 & 68.55 & 288 & 177 & 6.54 & 34.3 & 4.72 & 0.723628 & 14 & 0.1152 & 0.512034 & 9 & 0.712903 & -10.2 \\
\hline & 97GD61 & 69.03 & 267 & 221 & 6.08 & 29.0 & 3.49 & 0.723439 & 12 & 0.1269 & 0.512037 & 10 & 0.715507 & -10.3 \\
\hline & 97GD54 & 71.66 & 448 & 148 & 6.25 & 32.9 & 8.77 & 0.732235 & 11 & 0.1149 & 0.512020 & 12 & 0.712298 & -10.4 \\
\hline \multirow[t]{3}{*}{ Dadongshan } & $21 \mathrm{GD} 08$ & 72.00 & 364 & 54.6 & 10.7 & 61.1 & 19.3 & N.A. & & 0.1059 & 0.512003 & 12 & & -10.6 \\
\hline & $21 \mathrm{GD} 11$ & 75.10 & 539 & 8.78 & 7.28 & 20.3 & 178 & N.A. & & 0.2168 & 0.512136 & 11 & & -10.2 \\
\hline & 21GD07 & 77.43 & 356 & 26.8 & 7.76 & 32.2 & 38.5 & N.A. & & 0.1457 & 0.512065 & 12 & & -10.2 \\
\hline \multicolumn{15}{|c|}{ NLM gabbro and syenites $(T=173-136 \mathrm{Ma})$} \\
\hline Chebu & $99 \mathrm{CB} 02$ & 49.87 & 58.8 & 307 & 5.09 & 22.2 & 0.554 & 0.707825 & 13 & 0.1384 & 0.512569 & 13 & 0.706462 & $-0.1^{\mathrm{a}}$ \\
\hline Quannan & 99QN06 & 54.25 & 94.9 & 615 & 10.0 & 49.1 & 0.447 & 0.705864 & 22 & 0.1233 & 0.512715 & 11 & 0.704816 & $3.0^{\mathrm{b}}$ \\
\hline Paitan & 97GD77 & 62.14 & 135 & 236 & 10.3 & 54.8 & 1.65 & 0.710877 & 13 & 0.1142 & 0.512431 & 10 & 0.707492 & $-2.6^{\mathrm{c}}$ \\
\hline Ejinao & 99GD84 & 59.18 & 264 & 66.2 & 7.01 & 50.0 & 11.5 & 0.729378 & 12 & 0.0848 & 0.512472 & 9 & 0.707059 & $-1.3^{\mathrm{d}}$ \\
\hline
\end{tabular}

$\varepsilon \mathrm{Nd}(T)=\left[\left({ }^{143} \mathrm{Nd} /{ }^{144} \mathrm{Nd}\right)_{\text {sample }}(T)\right.$
$\times(\exp \lambda T-l), \lambda=0.00654 \mathrm{Ga}^{-1}$

N.A., not available due to low $\mathrm{Sr}$ content $(<50 \mathrm{ppm})$ or high $\mathrm{Rb} / \mathrm{Sr}$ ratio $(>5.0)$.

$\mathrm{a}$ and $\mathrm{b}$, age $=173$ and $165 \mathrm{Ma}$ (Li et al., 2003); c and d: age =145 and $136 \mathrm{Ma}$ (Hsieh et al., 2005). 

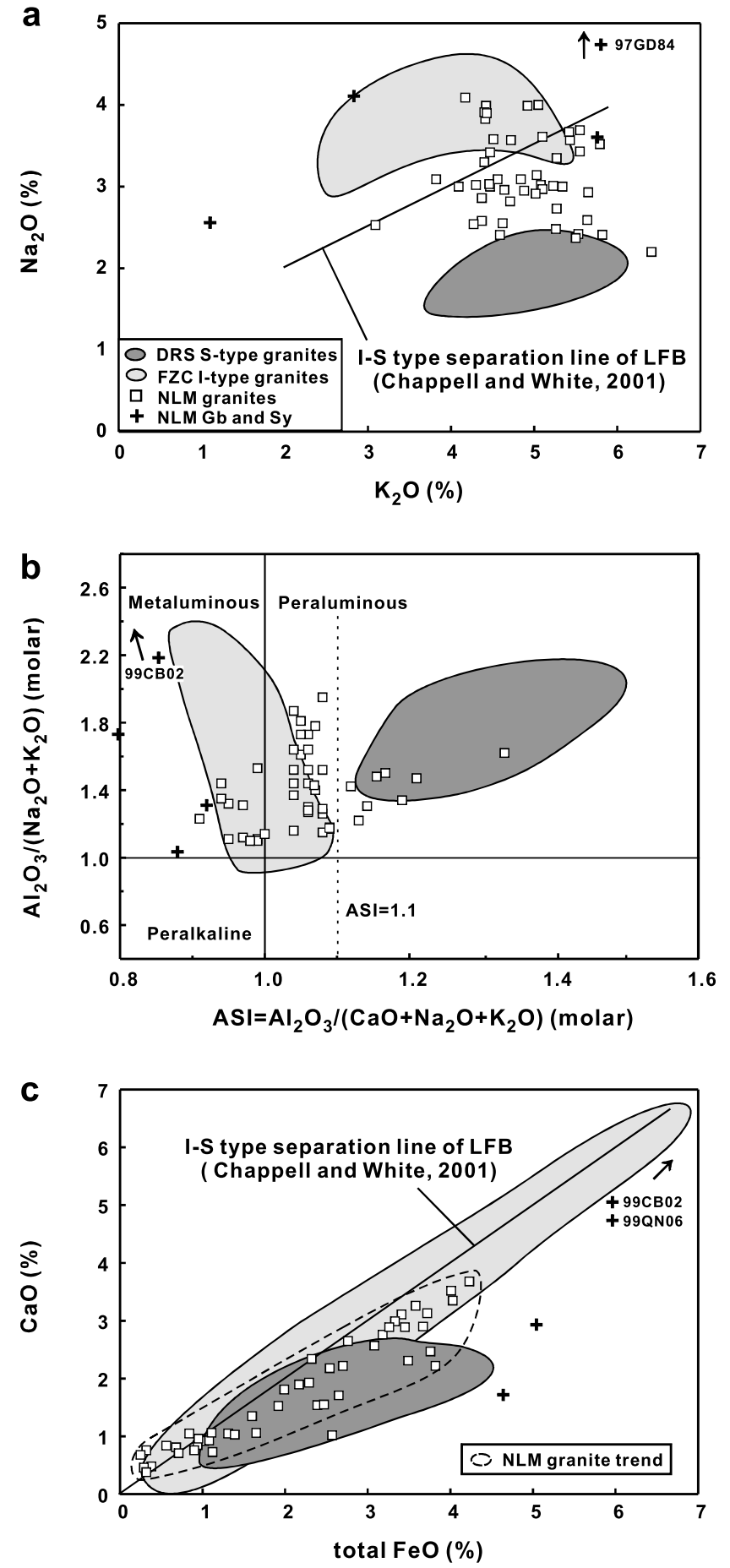

Fig. 2. (a) $\mathrm{Na}_{2} \mathrm{O}$ vs. $\mathrm{K}_{2} \mathrm{O}$ diagrams for the Mesozoic intrusive rocks from $\mathrm{S}$. China (b) $\mathrm{ANK}$ (molar $\mathrm{Al}_{2} \mathrm{O}_{3} /\left(\mathrm{Na}_{2} \mathrm{O}+\mathrm{K}_{2} \mathrm{O}\right)$ ) vs. $\mathrm{ACNK}$, or the aluminum saturation index (ASI) (molar $\left.\mathrm{Al}_{2} \mathrm{O}_{3} /\left(\mathrm{CaO}+\mathrm{Na}_{2} \mathrm{O}+\mathrm{K}_{2} \mathrm{O}\right)\right)$, and (c) $\mathrm{CaO}$ vs. total $\mathrm{FeO}$ (Table 1 and Appendix; Chen et al., 2000). DRS granites are strongly peraluminous (ASI > 1.1) and have low $\mathrm{CaO}$ and $\mathrm{Na}_{2} \mathrm{O}$ contents, whereas FZC granites are metaluminous to mildly peraluminous, and high in $\mathrm{CaO}$ and $\mathrm{Na}_{2} \mathrm{O}$, generally corresponding to the $\mathrm{S}$ - and I-type granites of the Lachlan Fold Belt (LFB), respectively. NLM granites have characteristics between DRS and FZC granites, while NLM gabbro and syenites have low aluminosity and various $\mathrm{CaO}$ and $\mathrm{Na}_{2} \mathrm{O}$ contents.

FZC granites - the I-type representatives of S. China (Fig. 4). However, granites differ markedly from gabbro

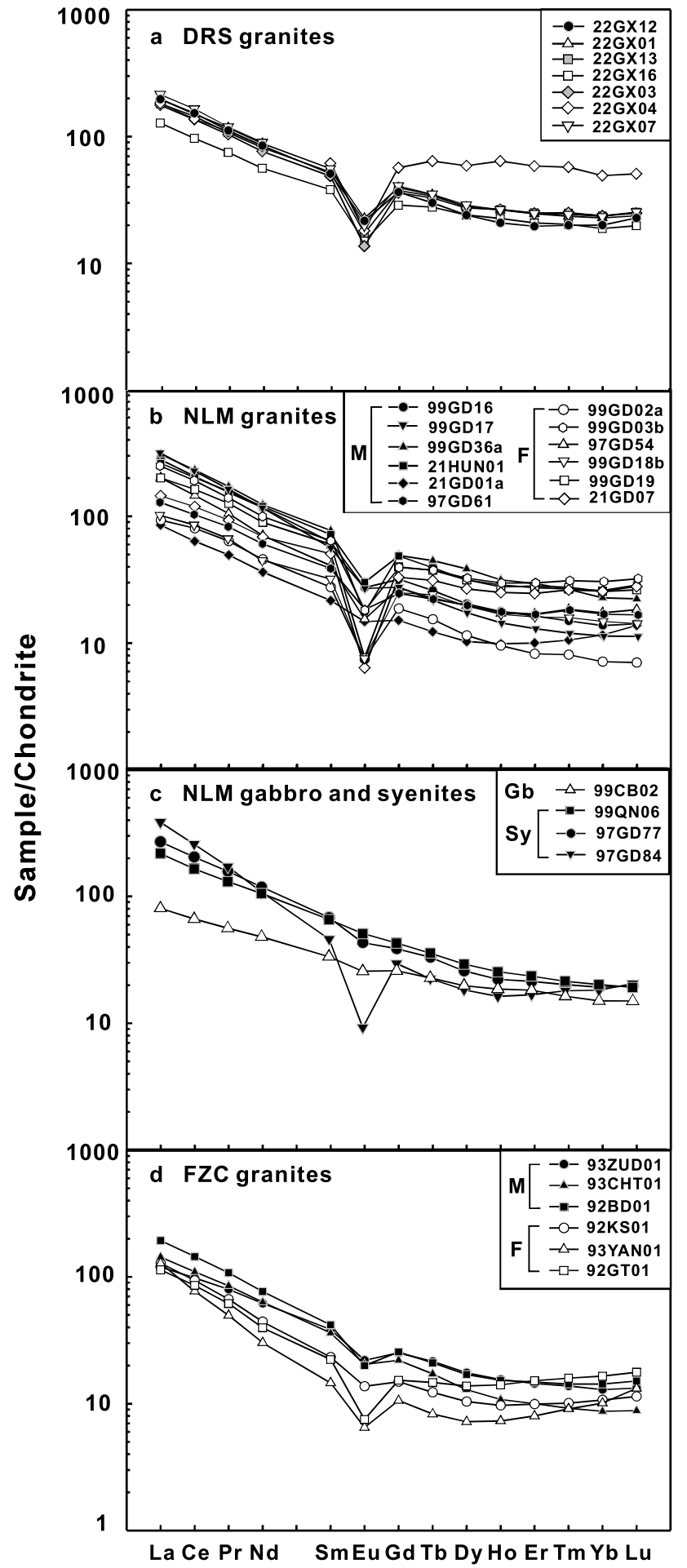

Fig. 3. Chondrite-normalized REE distribution patterns for (a) DRS granites, (b) NLM granites, (c) NLM gabbro and syenites, and (d) FZC granites of S. China. Normalizing values are those recommended by Sun and McDonough (1989). M, mafic granites $\left(\mathrm{SiO}_{2}=57-70 \mathrm{wt} \%\right) ; \mathrm{F}$, felsic granites $\left(\mathrm{SiO}_{2}>70 \mathrm{wt} \%\right)$. The $\mathrm{M}-\mathrm{F}$ division is that proposed by Sha and Chappell (1999).

and syenite in having more enriched $\mathrm{Sr}$ and $\mathrm{Nd}$ isotope compositions for the majority of samples $(\varepsilon \mathrm{Nd}(T)=-8.8$ to -11.6 and $\mathrm{Isr}=0.712-0.717)$. Few exceptions include (1) the Qitianling granodiorite (sample 21HUN01: 


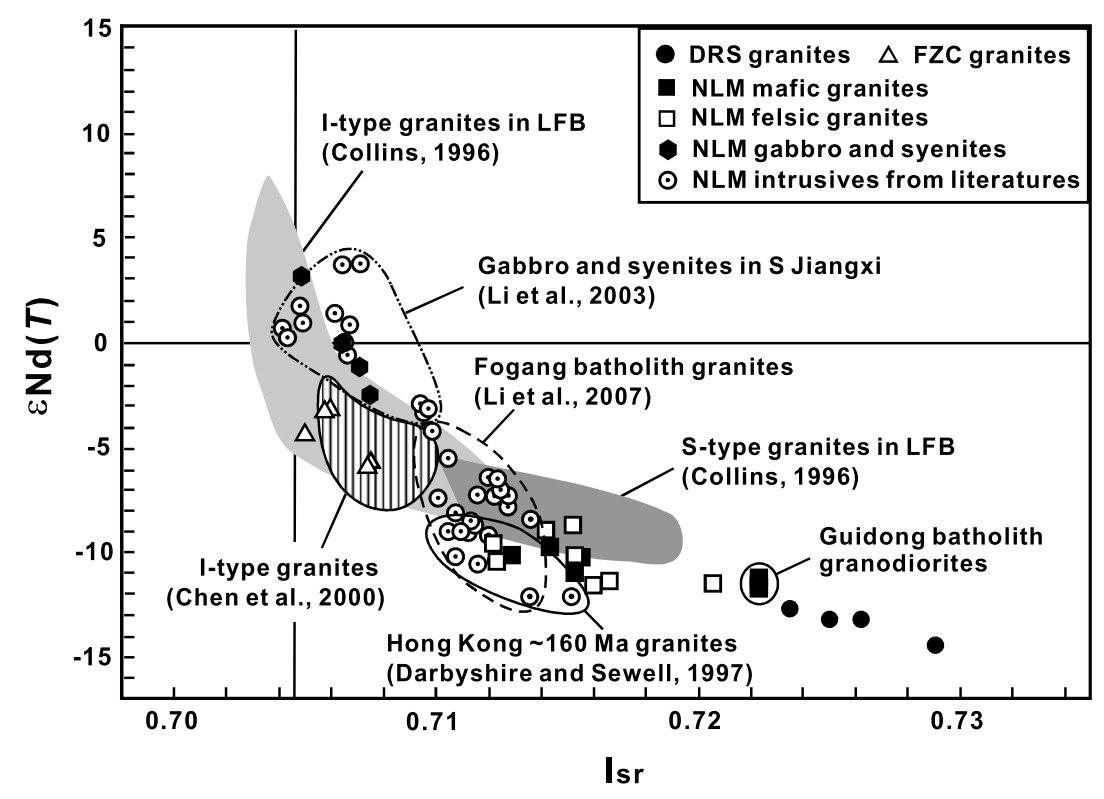

Fig. 4. $\varepsilon \mathrm{Nd}(T)$ vs. Isr plots for DRS, FZC, and NLM granites, and NLM gabbro and syenite in S. China (Table 2). They are compared with LFB S-type and I-type granites, gabbros, and syenites in S. Jiangxi, granites from Fogang batholith, and Hong Kong. Noted that many NLM mafic and felsic rocks are indistinguishable for $\mathrm{Sr}$ and $\mathrm{Nd}$ isotope compositions.

$\varepsilon \mathrm{Nd}(T)=-6.6$ and Isr is not available due to high $\mathrm{Rb} / \mathrm{Sr}$ ratio) that is close to FZC granites and (2) some Guidong granodiorites and granites $(\varepsilon \mathrm{Nd}(T)=-11.5$ to -12.4 and $\mathrm{Isr}=0.722)$ that are close to DRS granites. Our data for the Fogang granites $(\varepsilon \mathrm{Nd}(T)=-8.8$ to -11.6 and Isr $=0.712-0.721 ; n=10$, Table 2) show significantly higher Isr values than those $(n=17)$ reported by $\mathrm{Li}$ et al. (2007), indicating the complexity of magma sources for this huge batholith. Other NLM granites, although wide spreading in the Cathaysia Block, distribute in a small field somewhat deviated from that of the Fogang granites, but in large part coincide with the $\sim 160$ Ma Hong Kong granites to the south (Darbyshire and Sewell, 1997; $n=6$ ) (Fig. 4). Overall, the NLM granites, except Qitianling granodiorite, can be regarded as possessing intermediary $\mathrm{Sr}$ and $\mathrm{Nd}$ isotopic compositions between FZC and DRS granites.

\section{Apatite geochemistry}

Representative EMP and LA-ICP-MS analyses of apatites are shown in Tables 3 and 4, respectively. All these apatites are fluorapatites (averaged as $\mathrm{F}=3.2-5.4 \mathrm{wt} \%$ and $\mathrm{Cl}<0.3 \mathrm{wt} \%$ except sample 93ZUD01 that has $\mathrm{F}=3.1 \mathrm{wt} \%$ and $\mathrm{Cl}=1.0 \mathrm{wt} \%$ ), typical of igneous origin (Nash, 1984). Consistency of $\mathrm{F}$ and $\mathrm{Cl}$ contents among these apatites minimizes the possibility of being significantly affected by $\mathrm{F}$ metasomatism. Following the grouping of apatite geochemistry on LFB granites (Sha and Chappell, 1999), apatites are dealt separately with respect to the mafic $\left(\mathrm{SiO}_{2}=57-70 \mathrm{wt} \%\right)$ and felsic host rocks $\left(\mathrm{SiO}_{2}>70 \mathrm{wt} \%\right)$, except the DRS granites that are treated as a whole because of the small range of $\mathrm{SiO}_{2}(68-74 \mathrm{wt} \%)$.

\subsection{Sodium, silicon, and sulfur}

In contrast to the clear difference in the bulk $\mathrm{Na}_{2} \mathrm{O}$ contents (Fig. 2a), there is a large overlap on $\mathrm{Na}_{2} \mathrm{O}$ contents for apatites from DRS $\left(\mathrm{Na}_{2} \mathrm{O}=0.15-0.47 \mathrm{wt} \%\right)$ and FZC $\left(\mathrm{Na}_{2} \mathrm{O}=0.04-0.38 \mathrm{wt} \%\right)$ granites. However, the DRS apatites are distinct from FZC apatites for low $\mathrm{SiO}_{2}$ and $\mathrm{SO}_{3}$ contents (Fig. 5a and b). The $\mathrm{FZC}$ apatites display a positive $\mathrm{Na}_{2} \mathrm{O}-\mathrm{SO}_{3}$ correlation whereas the DRS apatites are characterized by low and relatively constant $\mathrm{SO}_{3}$ contents over a fourfold range in $\mathrm{Na}_{2} \mathrm{O}$ contents. These features resemble those shown by the LFB $\mathrm{S}-\left(\mathrm{SO}_{3}<\right.$ $0.05 \mathrm{wt} \%$ and $\mathrm{Na}_{2} \mathrm{O}>0.1 \mathrm{wt} \%$ ) and I-type granites $\left(\mathrm{SO}_{3}=0.03-0.75 \mathrm{wt} \%\right.$ and $\mathrm{Na}_{2} \mathrm{O}=0.02-0.15 \mathrm{wt} \%$ ) (Sha and Chappell, 1999). Overall, the NLM apatites have $\mathrm{Na}_{2} \mathrm{O}$ and $\mathrm{SiO}_{2}$ contents similar to FZC apatites, but have low $\mathrm{SO}_{3}$ contents that are the characteristics of DRS apatites. Therefore, based on the $\mathrm{Na}_{2} \mathrm{O}-\mathrm{SO}_{3}-\mathrm{SiO}_{2}$ relationship of the NLM apatites, their host granites cannot be explicitly assigned to S- or I-type.

\subsection{Thorium and uranium}

Thorium contents of the DRS apatites are the lowest (5-40 ppm) although the $\mathrm{U}$ concentrations are the highest (20-130 ppm) among all granitic suites. In contrast, FZC apatites have low $U$ concentrations $(<35 \mathrm{ppm})$ but high Th contents (10-90 ppm), and both are positively correlated. One mafic sample (93ZUD01) is exceptional for containing higher $U$ concentrations (Fig. 5c). Covariation between $U$ and Th of the NLM apatites mimics that of the FZC apatites, in which those from the felsic granites have low $\mathrm{Th}$ and $\mathrm{U}$ contents $(<35$ and $<20 \mathrm{ppm}$, respec- 
Table 3

Electron microprobe analyses in representative apatites from Darongshan (DRS), Fuzhou-Zhangzhou Complex (FZC), Nanling Mountains (NLM) granites, gabbro, and syenites of $\mathrm{S}$. China

\begin{tabular}{|c|c|c|c|c|c|c|c|c|c|}
\hline Locality & Sample No. (number) & $\mathrm{CaO}(\mathrm{wt} \%)$ & $\mathrm{P}_{2} \mathrm{O}_{5}(\mathrm{wt} \%)$ & $\mathrm{SiO}_{2}(\mathrm{wt} \%)$ & $\mathrm{Na}_{2} \mathrm{O}(\mathrm{wt} \%)$ & $\mathrm{SO}_{3}(\mathrm{wt} \%)$ & $\mathrm{F}(\mathrm{wt} \%)$ & $\mathrm{Cl}(\mathrm{wt} \%)$ & Total (wt \%) \\
\hline \multicolumn{10}{|l|}{ DRS granites } \\
\hline Jiuzhou & $22 \mathrm{GX} 12-5$ & 54.15 & 41.43 & 0.19 & 0.21 & 0.01 & 3.94 & 0.17 & 100.10 \\
\hline Taima & $22 \mathrm{GX} 01-2$ & 52.19 & 42.26 & 0.25 & 0.28 & 0.03 & 4.52 & 0.29 & 99.82 \\
\hline \multirow[t]{2}{*}{ Pubai } & $22 \mathrm{GX} 13-5$ & 54.55 & 41.39 & 0.17 & 0.19 & 0.02 & 4.52 & 0.03 & 100.87 \\
\hline & $22 \mathrm{GX} 16-2$ & 55.19 & 40.21 & 0.15 & 0.17 & 0.02 & 4.38 & 0.03 & 100.15 \\
\hline \multirow[t]{3}{*}{ Nadong } & $22 \mathrm{GX} 07-2$ & 53.35 & 42.55 & 0.27 & 0.25 & 0.03 & 4.11 & 0.08 & 100.64 \\
\hline & $22 \mathrm{GX} 03-3$ & 52.45 & 41.46 & 0.28 & 0.23 & $<0.01$ & 5.33 & 0.06 & 99.81 \\
\hline & $22 \mathrm{GX} 04-2$ & 51.16 & 43.32 & 0.25 & 0.41 & $<0.01$ & 3.60 & 0.09 & 98.83 \\
\hline \multicolumn{10}{|l|}{ FZC granites } \\
\hline Zudi & 93ZUD01-6 & 52.99 & 40.44 & 0.36 & 0.13 & 0.03 & 3.00 & 1.11 & 98.06 \\
\hline Changtai & 93СНТ01-8 & 54.33 & 40.69 & 0.42 & 0.11 & 0.08 & 4.55 & 0.25 & 100.43 \\
\hline Danyang & $92 \mathrm{BD} 01-3$ & 54.37 & 39.85 & 0.42 & 0.16 & 0.25 & 4.56 & 0.16 & 99.77 \\
\hline Fuzhou & $92 \mathrm{KS} 01-7$ & 52.55 & 40.86 & 0.56 & 0.20 & 0.16 & 4.73 & 0.14 & 99.20 \\
\hline Yanqian & 93YAN01-6 & 54.22 & 42.14 & 0.43 & 0.09 & 0.22 & 4.13 & 0.01 & 101.24 \\
\hline Lianjiang & 92GT01-3 & 53.12 & 40.45 & 0.48 & 0.26 & 0.42 & 4.87 & 0.02 & 99.62 \\
\hline \multicolumn{10}{|l|}{ NLM granites } \\
\hline \multirow[t]{2}{*}{ Guidong } & 99GD16-4 & 53.95 & 40.95 & 0.24 & 0.01 & 0.02 & 5.13 & 0.01 & 100.31 \\
\hline & 99GD18b-1 & 53.99 & 40.33 & 0.08 & 0.16 & $<0.01$ & 4.00 & $<0.01$ & 98.56 \\
\hline \multirow[t]{3}{*}{ Fogang } & 99GD36a-9 & 52.89 & 42.28 & 0.19 & 0.04 & 0.02 & 4.79 & 0.12 & 100.33 \\
\hline & 99GD02a-1 & 52.49 & 41.95 & 0.20 & 0.16 & 0.04 & 4.79 & $<0.01$ & 99.63 \\
\hline & 99GD19-3 & 53.03 & 40.60 & 0.52 & 0.16 & $<0.01$ & 5.41 & 0.01 & 99.73 \\
\hline Qitianling & 21HUN01-6 & 54.41 & 39.98 & 0.48 & 0.08 & 0.05 & 5.16 & 0.02 & 100.18 \\
\hline Lianyang & 21GD01a-1 & 54.15 & 39.77 & 0.48 & 0.09 & 0.03 & 5.10 & $<0.01$ & 99.62 \\
\hline Jiufeng & 97GD54-3 & 51.31 & 42.62 & 0.29 & 0.12 & $<0.01$ & 5.42 & 0.01 & 99.77 \\
\hline Dadongshan & $21 \mathrm{GD} 07-5$ & 52.27 & 40.45 & 0.66 & 0.09 & $<0.01$ & 5.03 & 0.01 & 98.51 \\
\hline \multicolumn{10}{|c|}{ NLM gabbro and syenites } \\
\hline Chebu & $99 \mathrm{CB} 02-2$ & 52.94 & 42.61 & 0.08 & 0.12 & $<0.01$ & 3.40 & 0.11 & 99.26 \\
\hline Quannan & 99QN06-4 & 53.12 & 38.57 & 0.63 & 0.25 & 0.20 & 5.13 & 0.14 & 98.04 \\
\hline Paitan & 97GD77-1 & 54.61 & 40.95 & 0.42 & 0.10 & 0.05 & 4.38 & 0.06 & 100.57 \\
\hline Ejinao & 97GD84-3 & 54.77 & 40.30 & 0.76 & 0.11 & 0.01 & 3.96 & 0.01 & 99.92 \\
\hline
\end{tabular}

tively) in spite that very few have slightly higher $\mathrm{U}$ contents. Low Th contents in the DRS apatites relative to others probably reflect the role of monazite, a Th-rich mineral that is rather abundant in the DRS granites (Chen et al., 2006). The possible mechanism for high U concentration in DRS apatites will be discussed later.

\subsection{Strontium and manganese}

DRS apatites generally contain less $\mathrm{Sr}$ and more $\mathrm{Mn}$ $(\mathrm{Sr}<85 \mathrm{ppm} ; \mathrm{Mn}=2100-8200 \mathrm{ppm})$ than FZC apatites $(\mathrm{Sr}=40-360 \mathrm{ppm} ; \mathrm{Mn}=550-4100 \mathrm{ppm})$, although there is an overlap between the fields for DRS and FZC apatites (Fig. 5d). Based on Mn content alone, FZC apatites can be distinguished between those forming in the maifc $(\mathrm{Mn}=550-1200 \mathrm{ppm})$ and felsic $(\mathrm{Mn}=1900-4100 \mathrm{ppm})$ rocks. Apatites from NLM granites apparently can be grouped for forming in the mafic (Mn $<800 \mathrm{ppm})$ and felsic $(\mathrm{Mn}>2100 \mathrm{ppm})$ rocks as well, and those from gabbro and syenites $(\mathrm{Mn}=230-1230 \mathrm{ppm})$ are close to the mafic rocks. In the case of LFB granites, low-Mn apatites $(<1000 \mathrm{ppm})$ only appear in mafic I-type granites that also contain more $\mathrm{Sr}(110-400 \mathrm{ppm})$ than apatites from S-type and felsic I-type granites (30-210 ppm) (Sha and Chappell, 1999). However, Sr content of apatites from NLM granites, varied from $55-200 \mathrm{ppm}$ in mafic to $30-160 \mathrm{ppm}$ in felsic rocks, is not as obvious as $\mathrm{Mn}$ content for discriminating these two groups of rock.

\subsection{REE (plus Y) abundances and ratios}

Although total REE abundances of DRS and FZC apatites are generally the same $(5000-14,000 \mathrm{ppm})$, their LREE ( $\mathrm{La}-\mathrm{Eu}$ ) and HREE (Gd-Lu) behave differently. Hence using the LREE/HREE ratio, it is possible to differentiate between DRS $(<3.5)$ and FZC (6.0-30) apatites (Fig. 6a). Similarly, it is possible to distinguish the variation of $\mathrm{Eu}$ and the $\mathrm{Sm} / \mathrm{Nd}$ ratio between DRS $(\mathrm{Eu}<7.0 \mathrm{ppm}$ and $\mathrm{Sm} / \mathrm{Nd}>0.30)$ and $\mathrm{FZC}(\mathrm{Eu}>11 \mathrm{ppm}$ and $\mathrm{Sm} / \mathrm{Nd}<0.23$ ) apatites (Fig. 6b). In addition, the dominance of $\mathrm{Y}$ (in place of Ho) in DRS ( $\mathrm{Y}=3000$ $6000 \mathrm{ppm} ; \mathrm{Ce}=1000-3400 \mathrm{ppm})$ whereas $\mathrm{Ce}$ in $\mathrm{FZC}$ ( $\mathrm{Y}=700-2700 \mathrm{ppm} ; \mathrm{Ce}=2500-6000 \mathrm{ppm})$ apatites is noted (Fig. 6c). Behavior of REE in apatites from FZC mafic and felsic granites are generally the same, except one felsic sample 92KS01 that shows a tendency towards DRS apatites (Fig. 6a-c).

Total REE concentrations are rather variable in apatites from NLM granites (1800-14,500 ppm) and gabbro plus syenite (3800-47,000 ppm). Except sample 99GD36a (a 
Table 4

LA-ICP-MS analyses of trace element abundances (ppm) in representative apatites from Darongshan (DRS), Fuzhou-Zhangzhou Complexes (FZC), Nanling Mountains (NLM) granites, gabbro, and syenites of $\mathrm{S}$. Chin

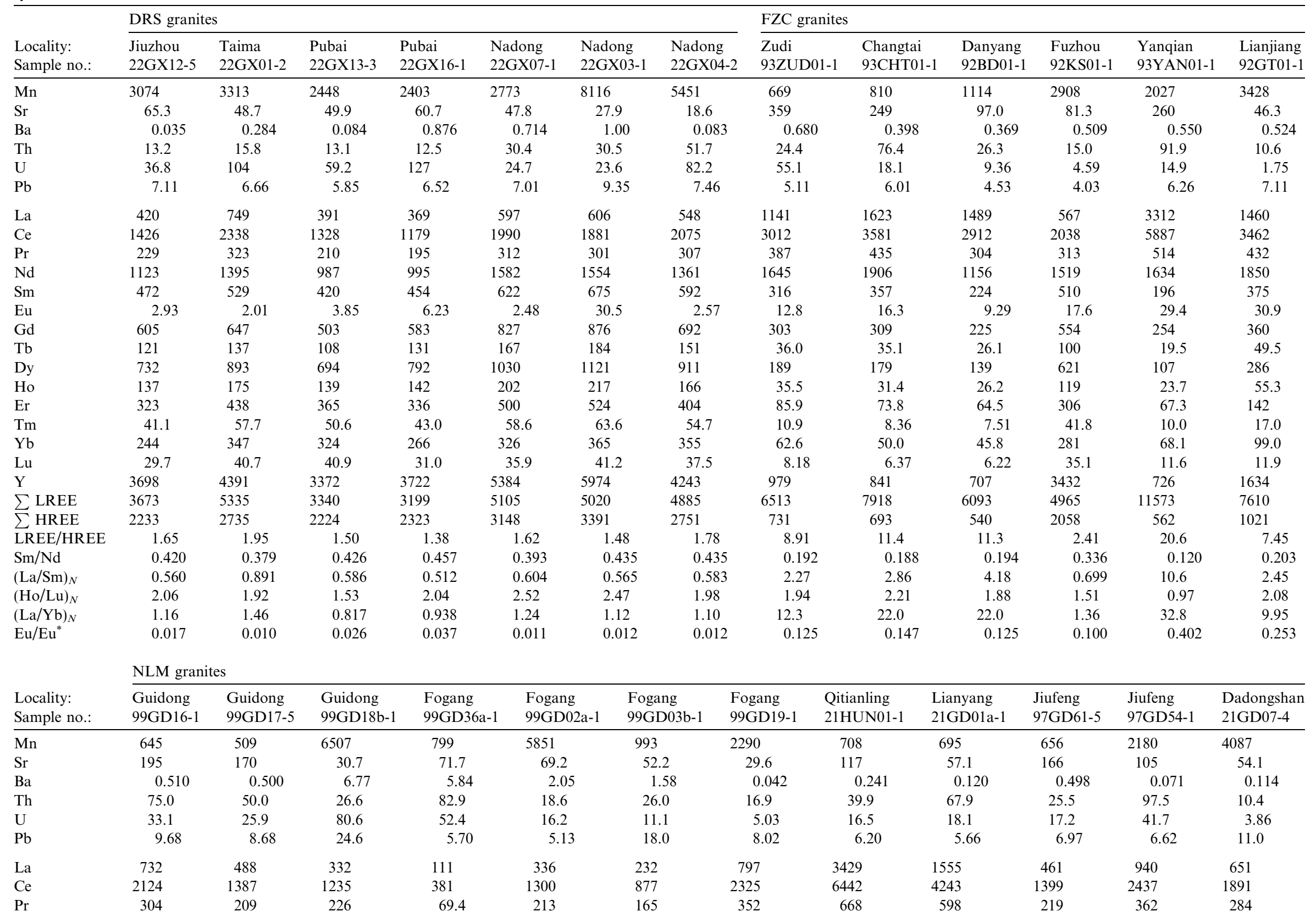




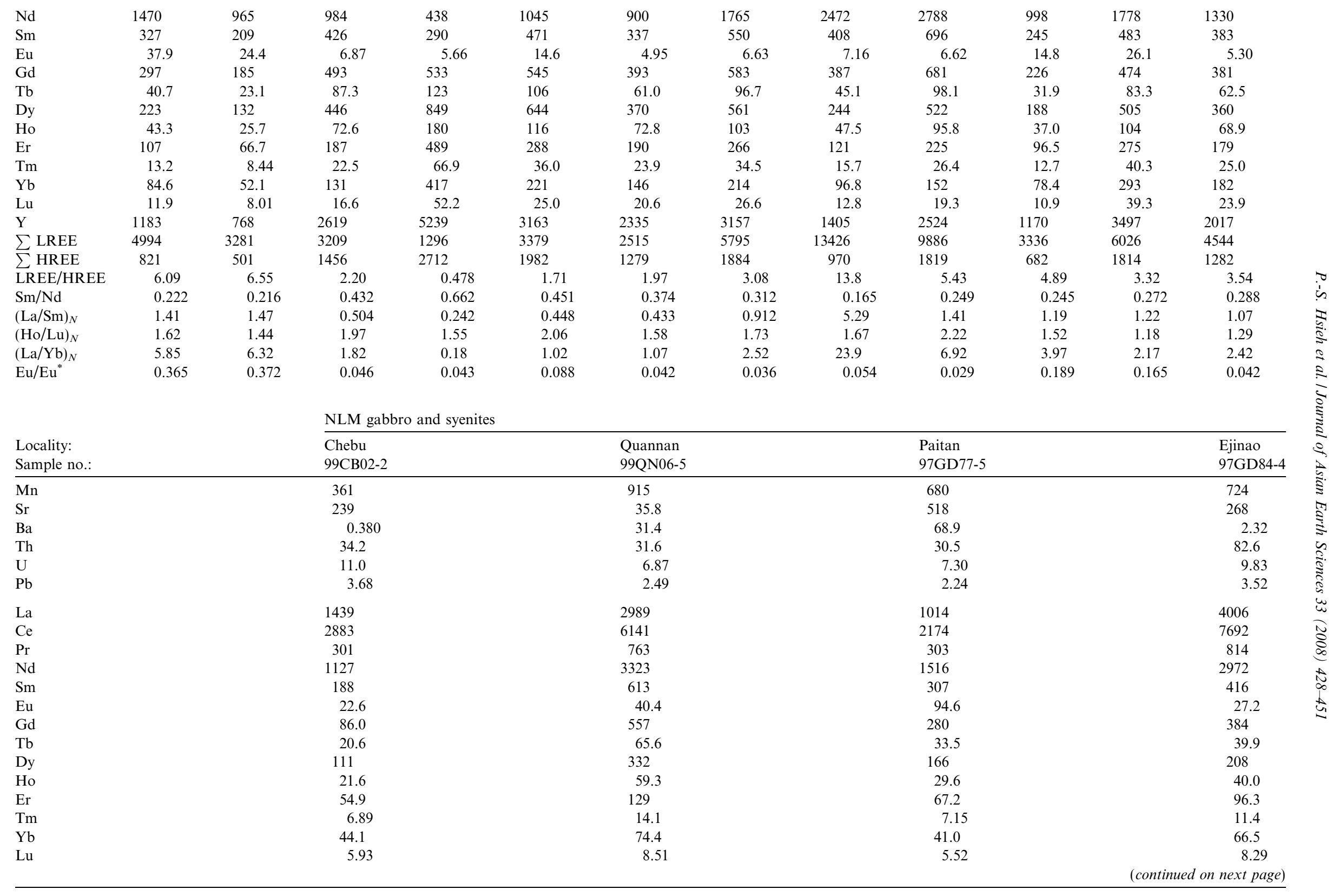




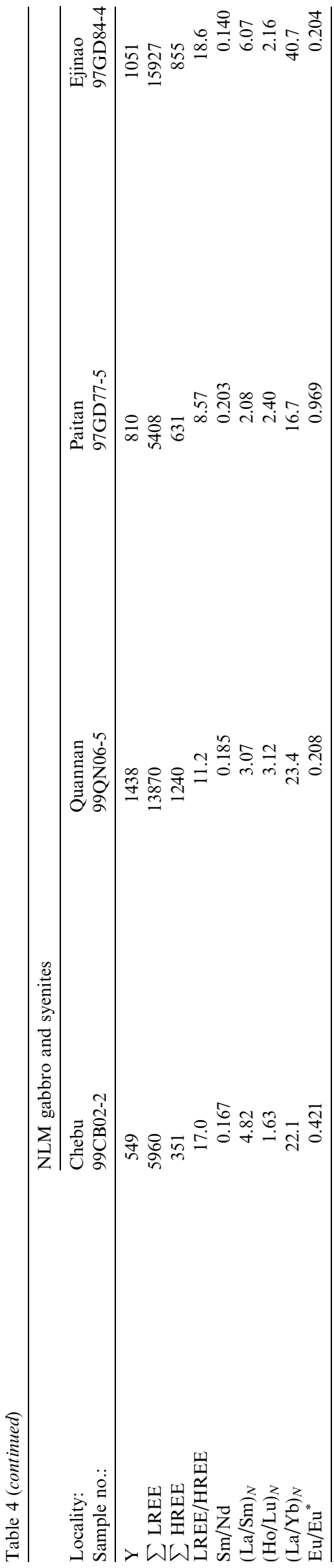

mafic pegmatitic granite), apatites from NLM mafic granites $\quad(\mathrm{LREE} / \mathrm{HREE}=4.0-14 ; \quad \mathrm{Eu}=4.7-45 \mathrm{ppm} ; \quad \mathrm{Sm} /$ $\mathrm{Nd}=0.16-0.28$ ) and gabbro plus syenite (LREE/ $\mathrm{HREE}=8.5-21 ; \quad \mathrm{Eu}=18-95 \mathrm{ppm} ; \quad \mathrm{Sm} / \mathrm{Nd}=0.13-0.21)$ have chemical characteristics similar to FZC apatites. In contrast, apatites from NLM felsic granites have intermediate chemical signatures between DRS and FZC apatites, i.e., slight enrichment in LREE (1600-6500 ppm) compared with HREE (900-2000 ppm), lower LREE/HREE (1.4-7.0), higher $\mathrm{Sm} / \mathrm{Nd}$ ratios (0.23-0.49) and enrichment in Y (Fig. 6a-c).

Apatites of sample 99GD36a are extremely low in total REE abundance $(<4000 \mathrm{ppm})$, especially LREE $(<1300 \mathrm{ppm})$ and $\mathrm{Eu}(<6 \mathrm{ppm})$, and LREE/HREE $(0.48$ 1.3), but high in HREE abundance, and $\mathrm{Sm} / \mathrm{Nd}$ ratio (0.46-0.66), with Y (>1300 ppm) more strongly enriched over $\mathrm{Ce}(<400 \mathrm{ppm})$ (Fig. 6a-c). Such features are in accord with those described for apatites from granite pegmatites (Belousova et al., 2002).

\subsection{Chondrite-normalized REE distribution patterns}

Apatites from DRS granites generally show very gentle convex or near-flat REE distribution patterns $((\mathrm{La} /$ $\mathrm{Sm})_{N}=0.47-1.17$ and $\left.(\mathrm{La} / \mathrm{Yb})_{N}=0.66-1.75\right)$ with strong negative $\mathrm{Eu}$ anomalies $\left(\mathrm{Eu} / \mathrm{Eu}^{*}<0.04\right)$. Like the case of LFB S-type granites (Sha and Chappell, 1999), a slight Nd depletion is observed (Fig. 7a). On the other hand, most apatites from FZC granites are characterized by straight and strongly right-inclined REE distribution patterns $\left((\mathrm{La} / \mathrm{Sm})_{N}=1.50-2.97\right.$ and $\left.(\mathrm{La} / \mathrm{Yb})_{N}=5.80-22.1\right)$ with weak to moderate $\mathrm{Eu}$ anomalies $\left(\mathrm{Eu} / \mathrm{Eu}^{*}=0.10\right.$ 0.30). Again these patterns resemble those of LFB I-type granites (Fig. 7b). However, unusual behavior is shown by apatites from fractionated I-type granites. One example is sample $93 \mathrm{YNC} 01$ which shows concave HREE patterns with low $(\mathrm{Ho} / \mathrm{Lu})_{N}$ ratios in apatites $(0.97-1.30)$, whereas the generalized straight and right-inclined LREE characteristics of apatites in this group of rocks are maintained (Fig. 7c). The host rock of these apatites shows a low $(\mathrm{Ho} / \mathrm{Lu})_{N}$ ratio $(0.55)$ as well (Fig. $\left.3 \mathrm{~d}\right)$, implying a linkage to the whole-rock geochemistry for such a concave HREE pattern of apatites. REE patterns of apatites from another fractionated sample $92 \mathrm{KS} 01$, which possesses the highest ASI value (1.04) in the FZC granites (Table 1), differ from the FZC I-type granite proper by having slightly rightinclined REE distribution patterns $\left((\mathrm{La} / \mathrm{Sm})_{N}=0.70-1.05\right.$ and $\left.(\mathrm{La} / \mathrm{Yb})_{N}=1.36-2.95\right)$ with moderate $\mathrm{Eu}$ anomalies $\left(\mathrm{Eu} / \mathrm{Eu}^{*}=0.10-0.14\right)$ (Fig. 7d). In fact, such REE patterns resemble apatites from the majority of NLM granites (see later sections).

Apatites from NLM granites show various kinds of REE patterns. Those from rocks with high ASI values $(\geqslant 1.1)$ are similar to DRS apatites, e.g., sample 99GD02a of the Fogang batholith and sample 99GD18b of the Guidong batholith (Fig. 7e), and those from rocks with low ASI values $(\leqslant 0.91)$ are similar to apatites from 

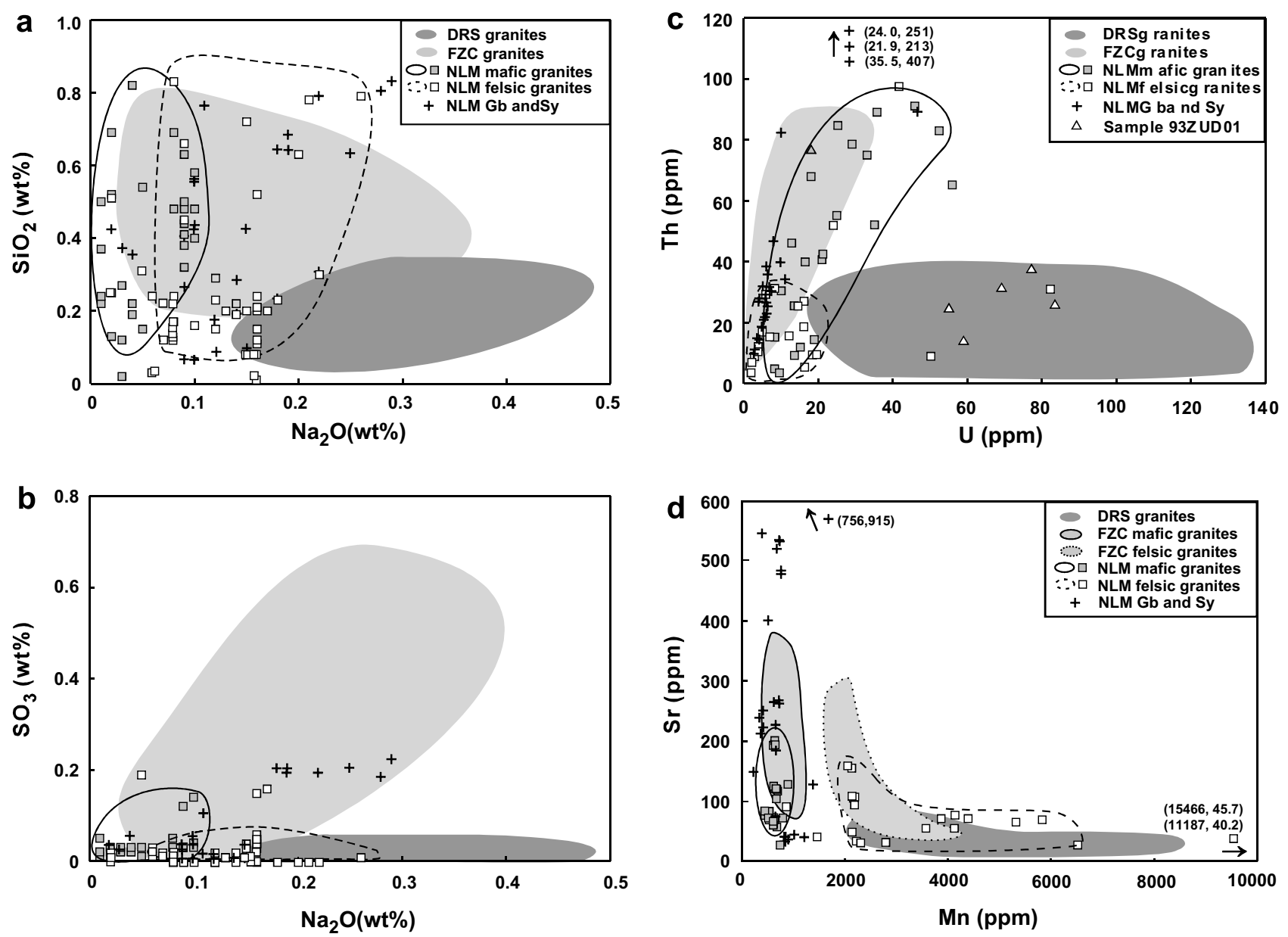

Fig. 5. (a) Si and Na concentrations, (b) S and Na concentrations in apatites from DRS, FZC, NLM granites, gabbro, and syenites of S. China. (c) Th and U concentrations, and (d) Sr and Mn concentrations in apatites from DRS, FZC, NLM granites, gabbro, and syenites of S. China.

the FZC granite proper, e.g., sample 21HUN01 (Qitianling batholith; Fig. 7f). In fact, the majority of apatites from NLM granites are metaluminous to mildly peraluminous $(\mathrm{ASI}=0.97-1.08)$ and characterized by slightly rightinclined REE distribution patterns $\left((\mathrm{La} / \mathrm{Sm})_{N}=0.40-1.68\right.$ and $\left.(\mathrm{La} / \mathrm{Yb})_{N}=0.76-10.9\right)$ with various $\mathrm{Eu}$ negative anomalies $\left(\mathrm{Eu} / \mathrm{Eu}^{*}=0.02-0.39\right)($ Fig. $7 \mathrm{~g}-\mathrm{j})$. They are distinguished clearly from NLM gabbro and syenites which are close to the FZC unfractionated I-type granite (Fig. 7k-n). A special type is shown by apatites from sample 99GD36a, the pegmatitic granite, that has strong depletion in LREE $\left((\mathrm{La} / \mathrm{Sm})_{N}=0.22-0.27\right)$ and variable HREE content $\left((\mathrm{La} / \mathrm{Yb})_{N}=0.18-0.67\right)$ plus moderate Eu anomalies $\left(\mathrm{Eu} / \mathrm{Eu}^{*}<0.06\right)$, leading to the unique convex apatite REE patterns comparable with those from Norwegian granite pegmatites (Fig. 7o).

\section{Discussion}

Trace element abundances of apatite often vary with some parameters of the host rock, such as the oxidation state, $\mathrm{SiO}_{2}$ content, total alkali and ASI values (Belousova et al., 2002). Here we examine the validity of these parameters (except total alkali) in relation to the geochemistry of apatites from different kinds of the Mesozoic granites in S. China. The effect of total alkali is not a major concern because it can be important only to the syenitic rocks which have very small population in the studied rocks that generally have calc-alkaline affinities.

\subsection{Preferential substitution of elements in apatite and oxidation state of magma}

In apatite, substitution of $\mathrm{Ca}^{2+}$ by trivalent lanthanides $\left(\mathrm{REE}^{3+}\right)$ and $\mathrm{Y}$ mainly involves charge compensated for smaller $\left(\mathrm{Si}^{4+}\right)$ and larger $\left(\mathrm{Na}^{+}\right)$atoms as expressed in the following reactions (Fleet and Pan, 1994; Sha and Chappell, 1999):

$\mathrm{LREE}^{3+}+\mathrm{Si}^{4+}=\mathrm{Ca}^{2+}+\mathrm{P}^{5+}$
$\mathrm{HREE}^{3+}+\mathrm{Na}^{+}=2 \mathrm{Ca}^{2+}$

The $\mathrm{Na}_{2} \mathrm{O}-\mathrm{SiO}_{2}$ relationship (Fig. 5a) and total LREE relative to HREE (Fig. 6a) for apatites from DRS strongly peraluminous granites, FZC and NLM metaluminous to slightly peraluminous granites, and NLM syenites and gabbro provide evidence to support the preference of reaction (2) substitution in the former and reaction (1) in the latter 

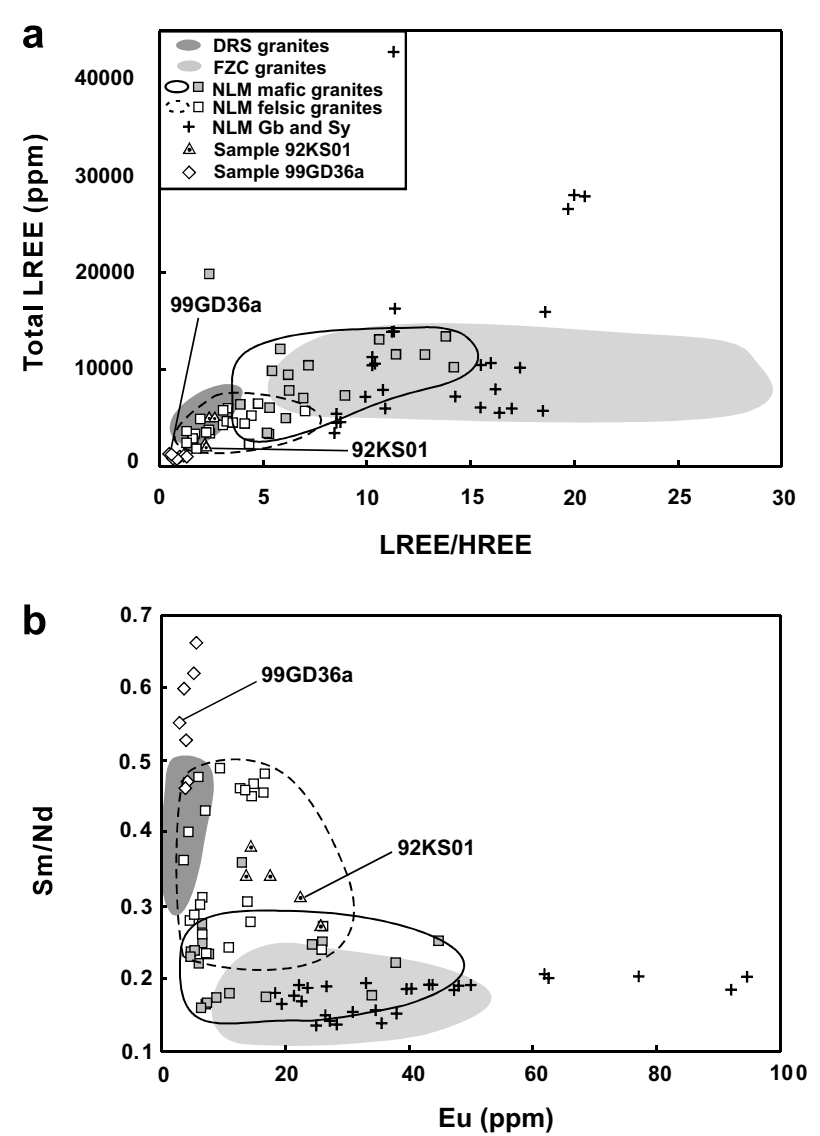

C

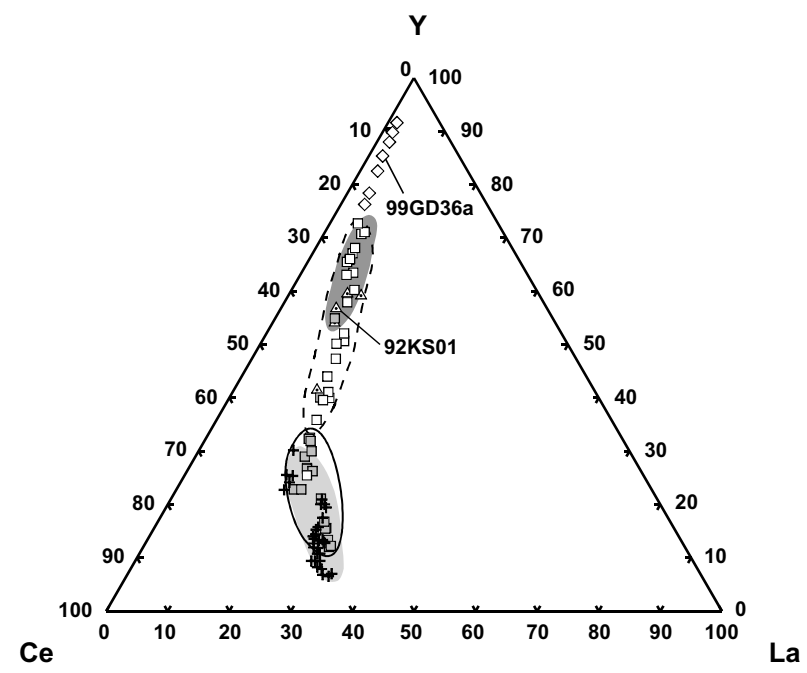

Fig. 6. (a) Total LREE concentration and LREE/HREE ratio plots, (b) $\mathrm{Sm} / \mathrm{Nd}$ ratio and Eu concentration plots and (c) Y-Ce-La triangular plots for apatites from DRS, FZC, NLM granites, gabbro, and syenites of S. China.

two kinds of rock. A few NLM felsic rocks that have ASI $>1.1$ also follow reaction (2).

The ionic state of $\mathrm{S}$ is another controlling factor for $\mathrm{Si}$ or $\mathrm{Na}$ enrichments in apatites: high oxygen fugacity conditions favor the presence of the $\mathrm{S}^{6+}$ state that can couple with $\mathrm{Na}^{+}$or $\mathrm{Si}^{4+}$ to substitute for $\mathrm{P}^{5+}$ or $\mathrm{Ca}^{2+}$, whereas low oxygen fugacity environments enhance conversion of $\mathrm{S}^{6+}$ to $\mathrm{S}^{2-}$ which is highly incompatible in apatites (Sha and Chappell, 1999). Apparently, substitution of $\mathrm{S}^{6+}$ is insignificant for DRS apatites (Fig. 5b), indicating the dominance of $\mathrm{S}^{2-}$ under the lower oxygen fugacity environment for strongly peraluminous magmas. On the contrary, the positive correlation between $\mathrm{SO}_{3}$ and $\mathrm{Na}_{2} \mathrm{O}$ in apatites from FZC granites supports the substitution of $\mathrm{S}$ and $\mathrm{Na}$ for $\mathrm{Ca}$ and $\mathrm{P}$ in more oxidizing metaluminous magmas. The $\mathrm{Na}$ and $\mathrm{S}$ relationship in apatites from NLM syenites and gabbro generally follows the trend of FZC apatites, but $\mathrm{SO}_{3}$ and $\mathrm{Na}_{2} \mathrm{O}$ contents in apatites from NLM granites are low. Only those from felsic rocks have higher $\mathrm{Na}_{2} \mathrm{O}$ contents up to the level of DRS granites.

Abundances of $\mathrm{Mn}, \mathrm{U}$, and $\mathrm{Eu}$ in apatites have also been proposed to be controlled by the oxygen fugacity of the melt (Belousova et al., 2001). Extensive Mn and U enrichment in DRS apatites (Figs. 5c-d) strongly indicates that their host magmas have been subject to low oxygen fugacity conditions. The presence of accessory uraninite $\left(\mathrm{UO}_{2}=86.7\right.$ $93.3 \mathrm{wt} \%, \mathrm{ThO}_{2}=3.1-6.9 \mathrm{wt} \%, \mathrm{PbO}=2.5-2.8 \mathrm{wt} \%$, and $\mathrm{Y}_{2} \mathrm{O}_{3}=0.3-0.8 \mathrm{wt} \%$ ) in DRS granites is also diagnostic. Conversely, mafic granites of FZC and NLM granites (gabbro and syenites as well) are representative of more oxidized rocks. Again, magmas of a few NLM felsic granites are more likely to be equivalents at lower oxygen fugacity (Fig. 5c). It is worth noting that high $\mathrm{U}$ concentrations in apatite from sample 93ZUD01 in the FZC granites (55-85 ppm) is also accompanied by exceptionally high $\mathrm{Cl}(1.1 \mathrm{wt} \%)$ and low $\mathrm{F}(3.0 \mathrm{wt} \%)$ contents (Table 3). Because the host rock is not particularly rich in $\mathrm{U}$ (Table 1), this enrichment could be due to earlier crystallization of apatite, rather than the presence of another U-retaining mineral (most probably zircon) in the melt.

Owing to large amounts of $\mathrm{Eu}^{2+}$ entering into the feldspar structure, reduced melts, usually high in $\mathrm{Eu}^{2+} / \mathrm{Eu}^{3+}$ ratios (Sha and Chappell, 1999), would hold a strong Eu negative anomaly in the enclosing apatites. This can explain the case of the DRS apatites. On the contrary, a high amount of $\mathrm{Eu}^{3+}$ (or lower $\mathrm{Eu}^{2+} / \mathrm{Eu}^{3+}$ ratio) in oxidized melts can assist $\mathrm{Eu}$ occupancy in the apatite structure because the ionic radius of $\mathrm{Eu}^{3+}$ is similar to $\mathrm{Ca}^{2+}$. Such a mechanism could be responsible for the slight to medium Eu negative anomalies in FZC and NLM apatites. On this ground, apatites from Paitan syenite (sample 99GD77) that have higher Eu concentrations (20-95 ppm) leading to very slight or even obscure Eu negative anomalies $\left(\mathrm{Eu} / \mathrm{Eu}^{*}=1.0-0.24\right)$ in the REE patterns (Fig. 71), could have crystallized from melts with higher oxygen fugacities as well. In summary, DRS apatites appear to have crystallized from relatively reduced magmas, whereas FZC and the majority of NLM apatites have crystallized from more oxidized magmas, except those in a few felsic granites that may have formed from magmas with lower oxygen fugacity similar to the DRS apatites.

\subsection{Effects of coexisting REE-rich accessory minerals}

Based on small variations of REE concentration and similar whole-rock REE distribution patterns for the 

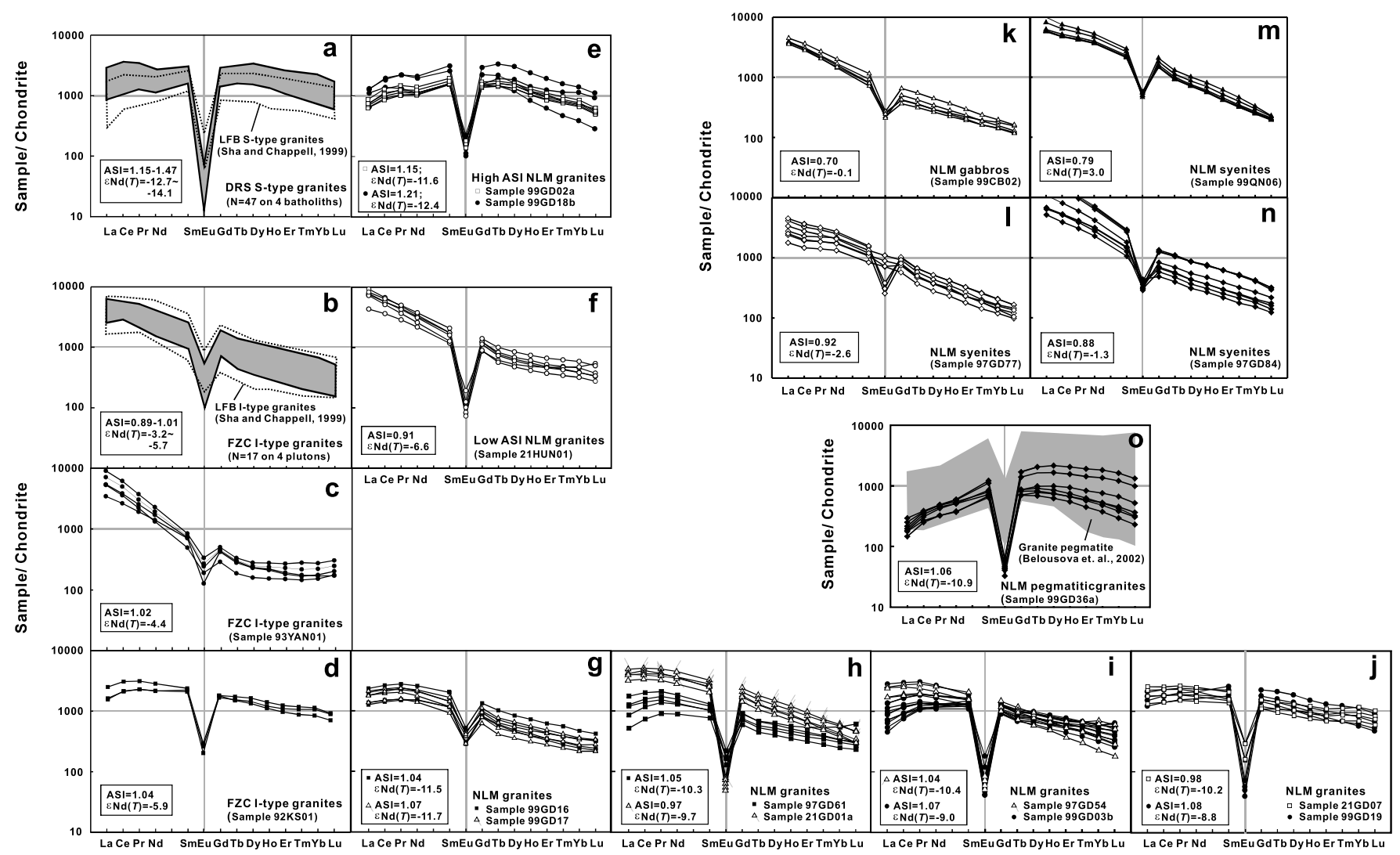

Fig. 7. Chondrite-normalized REE distribution patterns of apatites from (a) DRS and LFB S-type granites, (b-d) FZC granites (including LFB I-type granites in b), (e-j) NLM granites, ( $k-n)$ NLM gabbro and syenites, and (o) NLM pegmatitic granite. Normalizing values are after Sun and McDonough (1989).

Mesozoic granitoids in S. China (Fig. 3), large differences of REE abundances and distribution patterns present in their enclosing apatites are unlikely to be controlled by the whole-rock REE concentration. Therefore, internal redistribution of REE among different REE-rich minerals is considered more likely. Major factors are the mineral assemblage, crystallization sequence of REE-rich minerals, and partition coefficients of REE between the mineral and the melt. Among the common REE-retaining accessory minerals, apatite is high in all REE, zircon and xenotime are enriched in HREE, and monazite and allanite are characteristically enriched in LREE. Progressive replacement of allanite and sphene by monazite and xenotime commonly occur in rocks with increasing ASI and decreasing $\mathrm{CaO}$ (Bea, 1996).

Depletion of LREE in apatites from LFB S-type and felsic I-type granites has been ascribed to the crystallization of monazites which usually become saturated earlier than apatites in the low-Ca, reduced and strongly peraluminous magmas (Sha and Chappell, 1999). Generally, DRS granites and two NLM granitic samples (99GD02a and 99GD18b) possess the criteria of such magmas and indeed contain more abundant monazite (Montel et al., 1996; Chen et al., 2006) and allanite (Liu et al., 2005). The slight $\mathrm{Nd}$ negative anomaly for apatite from these rocks (Fig. 7a and e) matches early crystallization of monazite due to pre- dominance of $\mathrm{Nd}$, or the largest partitioning of $\mathrm{Nd}$ among REE, in this mineral (Charoy, 1986; Yurimoto et al., 1990).

In the high-Ca, more oxidized and metaluminous magma that usually forms I-type granites, monazite is absent. Chemical variations between mafic (granodiorite and monzogranite) and felsic (syenogranite and alkali feldspar granite porphyry) suites in the FZC granies mainly result from magmatic processes of crystal fractionation (Martin et al., 1994; Chen et al., 2000). Both apatite and zircon are considered to be early magmatic phases in the FZC granites and hence the REE composition of apatite may reflect a counterpart from zircon (Bea, 1996). That is why apatites from mafic and most felsic suites have the same right-inclined distribution patterns (Fig. 7b). In some cases (e.g., felsic rocks) the effect of predominant zircon fractional crystallization over apatite would result in concave-upward HREE distribution patterns (i.e., (Ho/ $\left.\mathrm{Lu})_{N}<1\right)$. This can appear not only in the host rocks (Fig. 3c), but also in the containing apatites (Fig. 7c).

Ward et al. (1992) reported that magma evolution controlled by crystal fractionation of plagioclase, biotite and accessory apatite, monazite, zircon and xenotime can lead to strong reduction of REE concentrations with $\mathrm{SiO}_{2}$ varying from 71 to $74 \mathrm{wt} \%$ in the Dartmoor granitic pluton (SW England). Basically, crystallization dominated either by LREE-rich monazite or by HREE-rich zircon 
fractionation at two different stages is responsible for such distinctions. Judging from the similarity of whole-rock REE concentrations and patterns within a wide range of silica contents $\left(\mathrm{SiO}_{2}=63-78 \mathrm{wt} \%\right)$ as well as the scarcity of monazite, xenotime, and allanite in the NLM granites, involvement of these accessory minerals during the course of magma evolution is very small, not even to affect the REE shape of coexisting apatites. In few cases, zircon crystallization indeed can cause the whole-rock HREE patterns to become concave upward (Fig. 3b), but not their enclosing apatites (Fig. $7 \mathrm{~g}-\mathrm{j}$ ).

The particular type of REE distribution patterns for apatites from NLM pegmatitic granites (sample 99GD36a; Fig. 7o) is considered to be the only case that has been significantly affected by crystallization of REErich accessory minerals. Petrographically, there are many large $(1.3-1.5 \mathrm{~mm})$ euhedral crystals of allanite and sphene in this rock, and apatites occur mainly as inclusions in the large biotite (Fig. 8). Early crystallization of allanite (and sphene to a lesser degree; Sawka et al., 1990) can account for LREE depletion in apatites, because partition coefficients of LREE between allanite and high-silica melt (Mahood and Hildreth, 1983) are very similar to those between monazite and peraluminous melt (Montel, 1986, 1993). In other words, allanite alone has the ability to cause a strong LREE depletion in later apatites for sample 99GD36a.

\subsection{Apatite REE distribution patterns and whole-rock silica contents, ASI and $\varepsilon N d(T)$ values}

The geochemistry of apatite seems to provide a tool for distinguishing mafic from felsic granites in the Nanling Mountains area. For example, $\mathrm{Na}, \mathrm{Mn}$, and REE contents of apatites from NLM felsic rocks are more akin to those from DRS granites (Figs. 5a and d and 6), that are felsic or near-felsic $\left(\mathrm{SiO}_{2}=68-75 \mathrm{wt} \%\right)$. Also, the apatite REE distribution pattern becomes more flattened, relative to gabbro and syenites (Fig. $7 \mathrm{k}-\mathrm{n}$ ), as the whole-rock $\mathrm{SiO}_{2}$ content increases (Fig. $7 \mathrm{~g}-\mathrm{j}$ ). Therefore, NLM granites seem to follow the general tendency that, when rocks become felsic in composition, their geochemical properties would be limited by the low-temperature melts (Chappell and White, 2001). Such apatite geochemical features may thus be related to the low crystallization temperature of magmas.

Furthermore, apatite REE geochemistry, especially the REE distribution patterns, varies concordantly with the ASI value of the host granites, even including gabbro and syenites. Apatites from NLM high ASI $(\geqslant 1.1)$ granites (e.g., samples 99GD02a and 99GD18b) are more akin to the DRS pattern (host rock ASI $\geqslant 1.15$ ); those from gabbro and syenite bodies and low ASI $(\leqslant 0.91)$ batholiths (e.g., Qitianling) are same as the FZC pattern (host rock ASI $\leqslant 1.04)$; others with ASI values in between $(0.97$ 1.08 ), such as Guidong (1.04-1.07), Lianyang (0.97), Jiufeng (1.04-1.05), Fogang (1.07-1.08), and Dadongshan
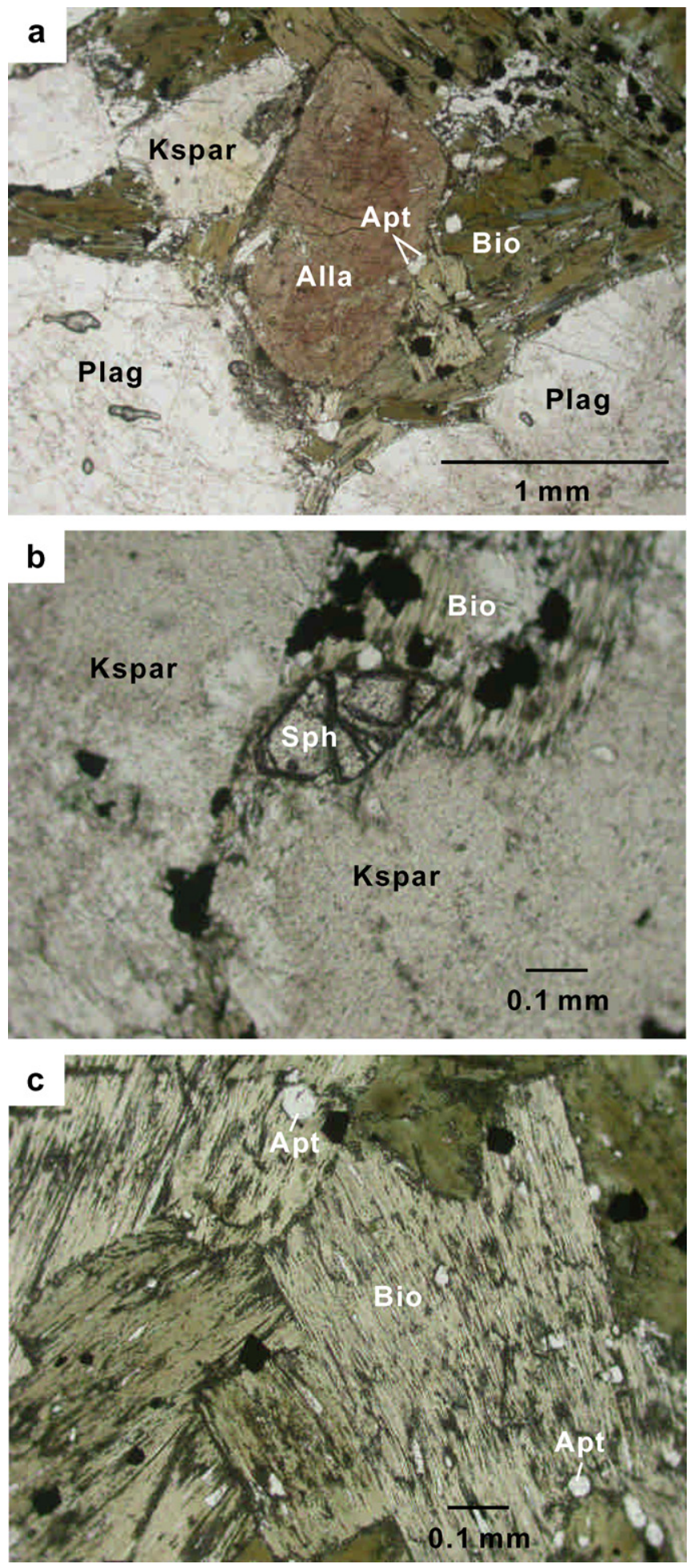

Fig. 8. (a) Photomicrograph showing the large euhedral allanite crystal in the NLM pegmatitic granite (sample 99GD36a). Small apatites crystallized in the boundary between allanite and biotite (open nicol) are noted (b) Photomicrograph showing the euhedral sphene crystal (open nicol). (c) Photomicrograph showing apatites occur mainly as inclusions in the large biotite (open nicol).

(0.98), are intermediary but closer to the FZC pattern (Fig. $7 \mathrm{~g}-\mathrm{j}$ ). In fact, this group of rocks are basically mildly peraluminous (ASI $=1.0-1.1$ ) varying in a wide range of 
silica $\left(\mathrm{SiO}_{2}=63-78 \mathrm{wt} \%\right)$. Moreover, the pegmatite sample (99GD36a), although having an ASI value (1.06) similar to this group, follows the unique apatite REE patterns of well-known pegmatitic granites elsewhere (Fig. 7o).

Isotopic composition is a useful parameter for indicating the source material of granitic magmas. Here we examine the REE distribution patterns of apatite from the studied rocks with their corresponding $\varepsilon \mathrm{Nd}(T)$ and ASI values. A striking feature is that samples with the characteristic apatite groups shown in Fig. 7 are clustered at the particular place on the $\varepsilon \operatorname{Nd}(T)$ vs. ASI diagram (Fig. 9). If this diagram is arbitrarily separated by two dividing lines on ASI $=1.1$ and $\varepsilon \operatorname{Nd}(T)=-8$, DRS granites and few NLM plutonic rocks (samples 99GD02a and 99GD18b) fall in the 4th quadrant (Group I); most NLM granodiorites and granites fall in the 3rd quadrant (Group IIa) with only Qitianling granodiorite (21HUN01) in the 2nd quadrant; FZC granites (Group IIIa) and NLM gabbro and syenites (Group IIIb), although all plotted in the 2nd quadrant, are classified into two groups according to rock type. On this basis, Qitianling granodiorite is thus included into Group IIIa. For the particular sample of the fractionated FZC granite (92KS01) that shows the same REE pattern of apatite as the majority of NLM rocks, it is further categorized as Group IIb by possessing high $\varepsilon \mathrm{Nd}(T)$ value.

Therefore, the majority of NLM granites, including both the mafic and felsic types $\left(\mathrm{SiO}_{2}=63-78 \mathrm{wt} \%\right)$, belong to Group IIa, suggesting that REE distribution patterns of their enclosing apatites are unlikely affected by the wholerock $\mathrm{SiO}_{2}$ contents, hence, the process of crystal fractionation. It is worthy to note that, although the Guidong granodiorites display Group IIa apatite patterns whereas the associated granite displays Group I patterns, they are plotted separately in the $\varepsilon \mathrm{Nd}(T)$-ASI space more akin to the NLM granite proper and DRS granites. Granitic rocks with higher ASI values favor crystallization of monazite the LREE phosphate in the magma (Chappell, 1999), coexisting apatites are thus depleted in LREE. Those which have ASI $<1.1$ need to be further examined for the $\varepsilon \mathrm{Nd}(T)$ value. Generally, the higher the $\varepsilon \mathrm{Nd}(T)$ value, the more enriched LEEE in the REE distribution pattern of apatites (Fig. 9). Conclusively, the case of NLM granites demonstrates that the host rock $\mathrm{ASI}$ and $\varepsilon \mathrm{Nd}(T)$ values, rather than $\mathrm{SiO}_{2}$ contents (or the mafic-felsic relationship) as suggested by Belousova et al. (2002), are the most sensitive parameters to correlate the shape of REE distribution in the enclosing apatites.

\subsection{Petrogenetic model of NLM granites}

Chappell, (1999) gave a thorough petrogenetic discussion on the I- and S-type granites in LFB based on the ASI values of these rocks. Major conclusions were (1) All the granites in the LFB are resulted from partial melting of the crust, with I-type rocks derived from low ASI $(<1.0)$ and S-type rocks from high ASI $(>1.0)$ sources; only their mafic suites can reflect the ASI of the sources. (2) Most mafic and felsic suites represent temperature-dependent partial melts, and felsic suites in I and $\mathrm{S}$ granite types may be indistinguishable due to similar compositions approaching the temperature minimal of granites. (3) If the mafic-felsic relationship is achieved through fractional crystallization, it shall lead to distinctly high ASI $(>1.1)$ in S-type granites, but close to 1.0 in I-type granites. How-

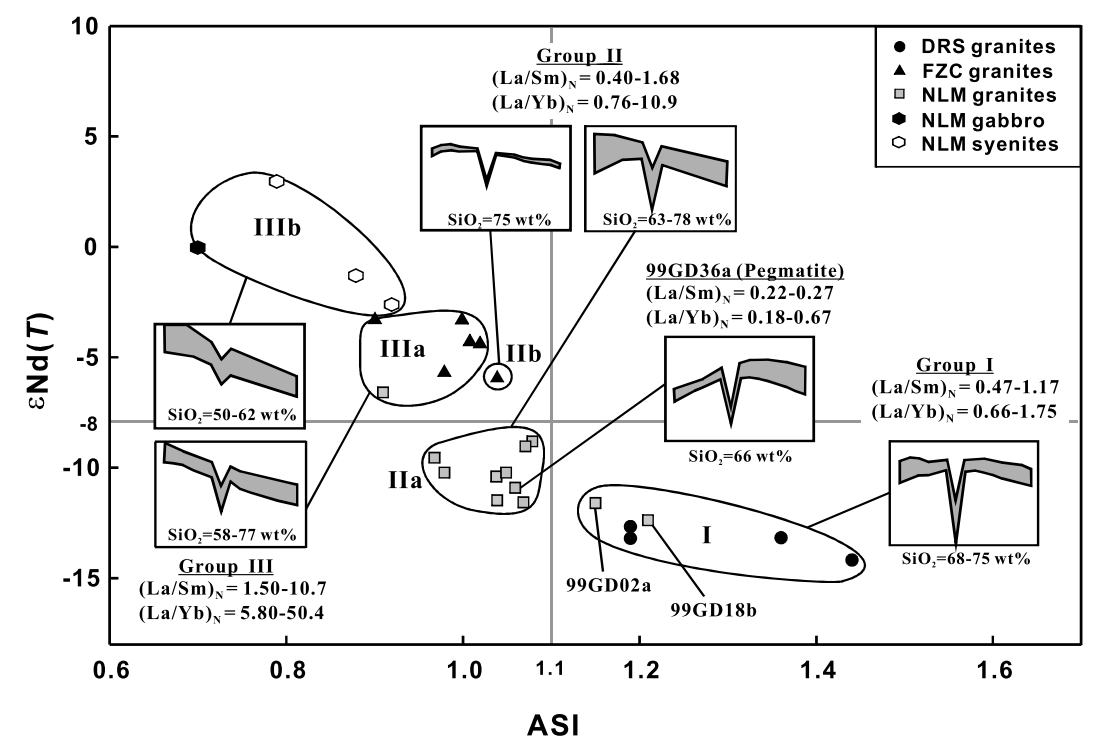

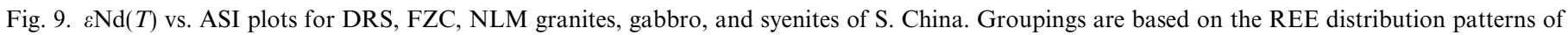

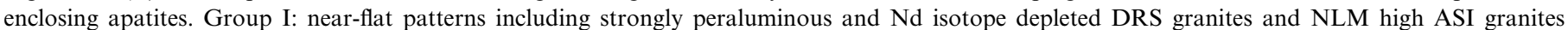

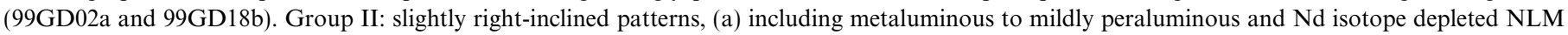

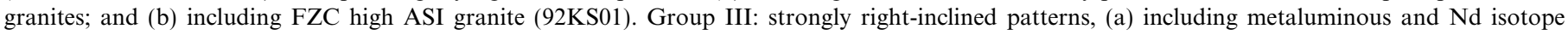

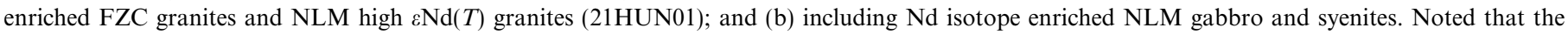
pegmatitic granite (99GD36a) shows unique patterns among all samples. 
ever, peraluminous compositions (ASI $>1.1$ ) caused by fractionation are inefficient and proposed to largely result from partial melting. (4) Behavior of some minor or trace elements, such as $\mathrm{P}$, helps to reveal the magmatic evolution of felsic suites in the two granite types. We use these criteria, together with grouping of the apatite REE pattern, to resolve Mesozoic granitoids in S. China.

Since both mafic (ASI < 1.0) and felsic (ASI 1.0) rocks of the FZC (Group IIIa) granites have ASI values $\leqslant 1$, along with the fact that $\mathrm{P}_{2} \mathrm{O}_{5}$ contents obviously decrease with the increasing $\mathrm{SiO}_{2}$ content (Table 1), felsic suites may be classified as typical I-type rocks through fractional crystallization from mafic suites. This is consistent with the scheme proposed by Martin et al. (1994) and Chen et al. (2000). On the other hand, DRS (Group I) granites can be either partial melting products from high ASI $(>1.1)$ sources, or highly fractionated rocks derived from mafic S-type suites. The latter process is less possible because of the lack of more mafic suites and the $\mathrm{P}_{2} \mathrm{O}_{5}-\mathrm{SiO}_{2}$ correlation among DRS granites (Table 1). As mentioned, the apatite geochemistry supports the idea of derivation of these rocks from more reducing sources.

Judging the results deduced form the apatite geochemisty and the fact that NLM granites (from mafic to felsic) are largely limited to Group IIa category, they are most probably derived from partial melting of more oxidized sources with ASI $=1.0-1.1$ under the higher and lower temperature conditions, respectively. Further evidenced from the relationship of Guidong granodiorite and granite, strongly peraluminous felsic NLM rocks $\left(\mathrm{SiO}_{2}=70.9\right.$ $73.5 \mathrm{wt} \%$; ASI $=1.15-1.21$ ) that match the Group I affinity with the characteristic REE distribution patterns of apatites (e.g., samples 99GD02a and 99GD18b) are more likely derived from different (more reducing) sources, or of the S-type origin because of the extremely high ASI values. Therefore, two separate crustal sources are considered to account for the origin of NLM granitic magmas, one may be equivalent to the Caledonian granites, and the other, the Indosinian granites. This postulation is based on the large population of these two ages (450-430 and 245-240 Ma) revealed from detrital monazites (Chen et al., 2006) and zircons (Xu et al., 2007) of some important drainage systems in the $\mathrm{S}$. China continent, which provides evidence for the prevalence of Caledonian and Indosinian basements in S. China.

Although magma generation for the vast NLM granites is still subject to much debate, there is a general consensus that these granites involve large amounts of crust material (Zhou and Li, 2000; Xu et al., 2005; Li et al., 2007). A simple assessment of the degree of crustal involvement is best shown by multi-component interactions between the crust and the mantle using $\mathrm{Sr}$ and $\mathrm{Nd}$ isotope compositions. The Caledonian crust is represented by its averaged composition C $(\mathrm{Sr}=295 \mathrm{ppm}$, $\mathrm{Nd}=50 \mathrm{ppm}$, Isr $=0.713$, and $\varepsilon \mathrm{Nd}(T)=-8.2$; Shen and Lin, 2002), the Indosinian crust is proposed to have a composition similar to sample $22 \mathrm{GX} 13$ of the DRS granitic suites $\mathrm{D}(\mathrm{Sr}=78 \mathrm{ppm}, \mathrm{Nd}=38 \mathrm{ppm}, \mathrm{Isr}=0.7284$, and $\varepsilon \operatorname{Nd}(T)=-14.1$; Table 2), and the most appropriate mantle component is the enriched source $\mathrm{M}(\mathrm{Sr}=$ $600 \mathrm{ppm}, \mathrm{Nd}=60 \mathrm{ppm}$, Isr $=0.7045$, and $\varepsilon \mathrm{Nd}(T)=+4.0)$ inferred from Li et al. (2003). To avoid the possible systematic errors, only our data with reliable Jurassic ages (Fig. 4) are used to test for the fitness of the mixing curve thus derived. The resultant curve for the two endmember components (M and D) has a curvature $(K)$ of 4.9 , as obtained from $(\mathrm{Sr} / \mathrm{Nd})_{\mathrm{M}} /(\mathrm{Sr} / \mathrm{Nd})_{\mathrm{D}}$. This curve, with $K$ shifts in a small range between 3.1 and 7.4, can match satisfactorily for all the NLM granites (Fig. 10). However, the mantle component is estimated to be around $20 \%$ for the majority of NLM granites which is in conflict with the Hf isotope data on zircons that suggest the dominance of an inheritance origin (Xu et al., 2005). Alternatively, because the averaged Caledonian-age granite $(\mathrm{C})$ is situated in this curve $(K=4.9)$ and close to the majority of NLM granites, a model involving melts derived from tectonothermal reactivation of predominant Caledonian and subordinate Indosinian granitic crusts would be more plausible.

The particular Group IIIa Qitianling granodiorite (sample 21HUN01) is positioned close to the component $\mathrm{C}$ in the C-M segment of the mixing curve (Fig. 10) based on the initial ${ }^{87} \mathrm{Sr} /{ }^{86} \mathrm{Sr}$ ratio $(0.709)$ for the $\mathrm{Rb}-\mathrm{Sr}$ isochron ( $\mathrm{Li}$ et al., 2006) and the $\varepsilon \mathrm{Nd}(T)$ value (-6.6, Table 2 ). Under the framework of regional crust melting, it could be regarded as the remelting product of pre-Meso-

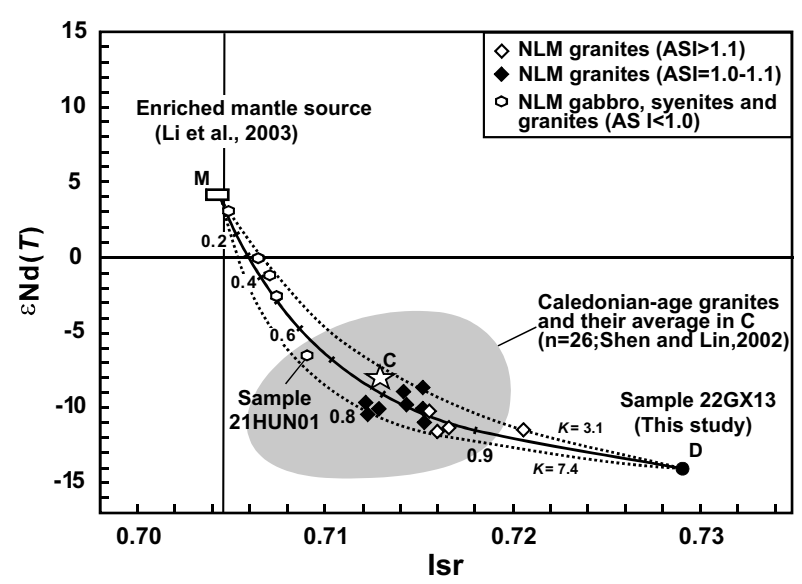

Fig. 10. Crust-mantle interactions using one end member of the Indosinian crust $\quad(\mathrm{Sr}=78 \mathrm{ppm} \quad$ and $\mathrm{Nd}=38 \mathrm{ppm} ; \quad \mathrm{Isr}=0.7284, \quad$ and $\varepsilon \mathrm{Nd}(T)=-14.1)$ represented by sample $22 \mathrm{GX} 13$ of DRS granites (D), and the other end member of an enriched mantle (M) source $(\mathrm{Sr}=600 \mathrm{ppm}$ and $\mathrm{Nd}=60 \mathrm{ppm} ; \mathrm{Isr}=0.7045$ and $\varepsilon \mathrm{Nd}(T)=+4)$ inferred from $\mathrm{Li}$ et al. (2003). The curve thus constructed has a curvature $(K)$ of 4.9, defined as $(\mathrm{Sr} / \mathrm{Nd})_{\mathrm{M}} /(\mathrm{Sr} / \mathrm{Nd})_{\mathrm{D}}$. Slight shift between $K=3.1-7.4$ can fit all the NLM granites. Large involvement of the crust component favors the model of in situ crust melting (Chen and Grapes, 2003), and sources are predominant Caledonian granites and subordinate Indosinian granites. The asterisk $\mathrm{C}$ denotes the averaged Caledonian-age granites (Shen and Lin, 2002). 
zoic granitic crust as well, but may have been affected by the mantle component to some extent. This is explained by the fact that Qitianling is situated in the Shi-Hang zone (Fig. 1a), a high $\varepsilon \mathrm{Nd}(T)$ magmatic belt coinciding with some Mesozoic rift basins (Gilder et al., 1996). The obvious extensional tectonic setting has rendered the Qitianling batholith unique in the ASI value, $\mathrm{Sr}$ and $\mathrm{Nd}$ isotope compositions as well as REE distribution patterns of apatite relative to other granitic intrusives in the Nanling Mountains area. Altogether, there seems to have a tendency that NLM rocks displaying higher ASI values also contain larger amounts of the component D (Fig. 10). This provides further supportive evidence of ASI-dependent magma genesis for granitic rocks (Chappell, 1999).

So far there are two main thoughts regarding the tectonic environment of NLM granites. One suggests that all the Mesozoic igneous rocks in S. China were generated through a flat subduction over a long distance and endured for a long span of time, under which NLM granites were related to the back-arc extension (Zhou and $\mathrm{Li}$, 2000) or slab foundering to explain the relaxation of a continental lithosphere ( $\mathrm{Li}$ et al., 2004; Li and Li, 2007). The other favors that NLM granites were the products of in situ melting or anatexis (Chen and Grapes, 2003) or via repeated remelting of the Proterozoic protolith based on Hf isotope compositions of the containing zircons (Xu et al., 2005). Now the REE distribution patterns of enclosing apatite in the NLM granites reflect that the great majority of these rocks must have contained predominantly crust materials and their $\mathrm{Sr}$ and $\mathrm{Nd}$ isotope compositions suggest that they are similar to the Caledonian and Indosinian granites. Therefore, we advocate that in situ crustal melting is the most plausible model to account for the genesis of Jurassic NLM granites (Groups IIa and I rocks). The existence of sporadic gabbro and syenite (Group IIIb rocks) and other relatively high $\varepsilon \mathrm{Nd}(T)$ granites (Group IIIa rocks) indicates local mantle input at places where crustal extension prevailed. This explains the heterogeneity of rock types and complexity of huge batholiths like Fogang, which may have involved mixing/mingling of the $\mathrm{D}, \mathrm{C}$, and $\mathrm{M}$ components shown in Fig. 10. Extensional or rifting tectonism causing uprising of mantle-derived magmas to underplate beneath the crust can provide the heat source for remelting of overlying crust materials. However, considerably more detail, including why the heat pulse for generating such a vast granitic terrain was largely limited to a short period of time $(160 \pm 5 \mathrm{Ma})$, is necessary to support this in situ melting model.

\section{Conclusions}

Apatite geochemistry has been suggested as a sensitive indicator of the crystallization environment and the distribution of trace elements in apatite can, therefore, be used to distinguish S-type and I-type granites. Although such an application to the Triassic DRS and Cretaceous FZC granites in S. China is generally valid, it is less applicable to the intermediary type Jurassic NLM granites. Our data reveal that the majority of NLM granites are metaluminous to mildly peraluminous (ASI values around 1.01.1), unaccompanied by monazite and allanite, and formed under relatively oxidizing (high oxygen fugacity) conditions more akin to I-type FZC granites. A few others are strongly peraluminous (ASI greatly $>1.1$ ) and probably formed under more reducing conditions similar to the Stype DRS granites. A striking feature is that REE distribution patterns of apatite strongly depends on the host rock ASI value, and the majority of NLM apatites can define a kind of pattern that is distinguishable from DRS and FZC apatites, or LFB S- and I-type apatites. This is a reflection of their source regions that are characterized by having specific ASI values, i.e., 1.0-1.1 vs. greatly $>1.1$. Furthermore, $\mathrm{Sr}$ and $\mathrm{Nd}$ isotope evidence indicates that two possible crustal sources for NLM granitic magmas can be represented predominantly by the pre-Mesozoic (mainly Caledonian) and subordinately by the pre-Yanshanian (mainly Indosinian) granitic rocks. We suggest that the most plausible petrogenetic model would be remelting of crustal materials composed mainly of these two precedent granitic rocks. Some NLM gabbro and syenitic rocks may represent mantle-derived melts emplaced during the relaxation of the continental lithosphere. These may play a more important role as the heat supplier that underplated beneath the crust, rather than as a geochemical modifier during the Jurassic crustal evolution in South China. Apatite REE geochemistry has been shown to be a useful tool to probe the petrogenesis of granites in the Nanling Mountain area of South China. The fact that grouping of apatite REE distribution patterns falls in different quadrants separated by $\mathrm{ASI}=1.1$ and $\varepsilon \mathrm{Nd}(T)=-8$ of the whole-rock analysis provides an alternative scheme of distinguishing source contributions for NLM granites.

\section{Acknowledgements}

The authors are indebted to Dr. Han-Wen Zhou of Faculty of Earth Sciences, China University of Geosciences (Wuhan), China for his guidance in the field, and Drs. S. Gao and X.M. Liu of same university and Key Laboratory of Continental Dynamics, Department of Geology, Northwest University (Xi'an), China for their arrangement for apatite analysis. The authors are also thankful to Prof. Xinmin Zhou and an anonymous reviewer for their constructive suggestions and comments on the manuscript. This paper is supported by research grants from the National Science Council, ROC (NSC91-2116-M002-032/Chen).

\section{Appendix A. Supplementary data}

Supplementary data associated with this article can be found, in the online version, at doi:10.1016/ j.jseaes.2008.02.002. 


\section{References}

Barbarin, B., 1999. A review of the relationships between granitoid types, their origins and their geodynamic environments. Lithos 46, 605-626.

Bea, F., 1996. Residence of REE, Y, Th and U in granites and crustal protoliths: Implication for the chemistry of crustal melts. Journal of Petrology 37, 521-552.

Belousova, E.A., Griffin, W.L., O'Reilly, S.Y., Fisher, N.I., 2002. Apatite as an indicator mineral for mineral exploration: trace-element compositions and their relationship to host rock type. Journal of Geochemical Exploration 76, 45-69.

Belousova, E.A., Walters, S., Griffin, W.L., O'Reilly, S.Y., 2001. Trace element signatures of apatites from granitoids of Mount Isa Inlier, north-west Queensland, Australia. Australian Journal of Earth Sciences 48, 603-619.

Chappell, B.W., 1999. Aluminum saturation in I- and S-type granites and the characterization of fractionated haplogranites. Lithos 46, 535-551.

Chappell, B.W., White, A.J.R., 1974. Two contrasting granite types. Pacific Geology 8, 173-174.

Chappell, B.W., White, A.J.R., 2001. Two contrasting granite types: 25 years later. Australian Journal of Earth Sciences 48, 489-499.

Charoy, B., 1986. The genesis of the Cornubian Batholith (Southwest England): the example of the Carnmenellis pluton. Journal of Petrology 27, 571-604.

Chen, C.-H., Lin, W., Lan, C.Y., Lee, C.Y., 2004. Geochemical, Sr and $\mathrm{Nd}$ isotopic characteristics and tectonic implications for three stages of igneous rock in the Late Yanshanian (Cretaceous) orogeny, SE China. Transaction Royal Society of Edinburgh: Earth Science 95, 237-248.

Chen, C.-H., Lin, W., Lu, H.Y., Lee, C.Y., Tien, J.L., Lai, Y.H., 2000. Cretaceous fractionated I-type granitoids and metaluminous A-type granites in SE China: the Late Yanshanian post-orogenic magmatism. Transaction Royal Society of Edinburgh: Earth Science 91, 195-205.

Chen, C.-H., Lu, H.Y., Lin, W., Lee, C.Y., 2006. Thermal event records in SE China coastal areas: constraints from monazite ages of beach sands from two sides of the Taiwan strait. Chemical Geology 231, 118-134.

Chen, C.-H., Tung, T.C., 1984. On-line data reduction for electron microprobe analysis. Acta Geologica Taiwanica 22, 196-200.

Chen, G., Grapes, R., 2003. An in-situ melting model of granite formation: geological evidence from southeast China. International Geology Review 45, 611-622.

Chen, J.F., Jahn, B.M., 1998. Crustal evolution of southeastern China: Nd and $\mathrm{Sr}$ isotopic evidence. Tectonophysics $284,101-133$

Clemens, J.D., 2003. S-type granitic magmas-petrogenetic issues, models and evidence. Earth Science Review 61, 1-18.

Collins, W.J., 1996. Lachlan Fold Belt granitoids: products of threecomponent mixing. Transaction Royal Society of Edinburgh: Earth Science 87, 171-181.

Darbyshire, D.P.F., Sewell, R.J., 1997. Nd and Sr isotope geochemistry of plutonic rocks from Hong Kong: implications for granite petrogenesis, regional structure and crustal evolution. Chemical Geology 143, 8193.

Deng, X.G., Chen, Z.G., Li, X.H., Liu, D.Y., 2004. SHRMP U-Pb zircon dating of the Darongshan-Shiwandashan granitoid belt in Southeastern Guangxi, China. Geological Review 50 (4), 426-432 (in Chinese with English abstract).

Fleet, M.E., Pan, Y.M., 1994. Site preference of $\mathrm{Nd}$ in fluorapatite $\left[\mathrm{Ca}_{10}\left(\mathrm{PO}_{4}\right)_{6} \mathrm{~F}_{2}\right]$. Journal of Solid State Chemistry $111,78-81$.

Frietsch, R., Perdahl, J.A., 1995. Rare earth elements in apatite and magnetite in Kiruna-type iron ores and some other iron ore types. Ore Geology Reviews 9, 489-510.

Frost, C.D., Bell, J.M., Frost, B.R., Chamberlain, K.R., 2001. Crustal growth by magmatic underplating: isotopic evidence from the northern Sherman batholith. Geology 29, 515-518.

Gao, S., Liu, X.M., Yuan, H.L., Hattendorf, B., Günther, D., Chen, L., Hu, S.H., 2002. Determination of forty two major and trace elements in USGS and NIST SRM glasses by laser ablation-inductively coupled plasma-mass spectrometry. Geostandard Newsletter 26, 181-196.
Gilder, S.A., Gill, J., Coe, R.S., 1996. Isotopic and paleomagnetic constraints on the Mesozoic tectonic evolution of south China. Journal of Geophysical Research 101 (B7), 16137-16154.

GRGNP (The Granitoid Research Group of the Nanling Project), 1989. Geology of granitoids of Nanling region and their petrogenesis and mineralization. Geological Publishing House, Beijing (in Chinese with English abstract).

Hsieh, P.S., Chen, C.-H., Yang, H.J., Lee, C.Y., Zhou, H.W., 2005. Geochronology and geochemistry of the Fogang granite-metaluminous A-type granite-syenite suite, Guangdong Province, S. China: implications for the petrogenesis of Early Yanshanian (Jurassic) intrusive rocks (Abstract). Structure, Tectonics and Ore Mineralization Processes, Townsville, Australia, p. 180.

Ishihara, S., 1977. The magnetite-series and ilmenite-series granitic rocks. Mining Geology 27, 293-305.

Jahn, B.M., Chen, P.Y., Yen, T.P., 1976. Rb-Sr ages of granitic rocks in southeastern China and their tectonic significance. Geological Society of America Bulletin 86, 763-776.

Jahn, B.M., Zhou, X.H., Li, J.L., 1990. Formation and tectonic evolution of Southeastern China and Taiwan. Isotopic and geochemical constraints. Tectonophysics 183, 145-160.

Lee, C.Y., Tsai, J.H., Ho, H.H., Yang, T.F., Chung, S.L., Chen, C.-H., 1997. Quantitative analysis in rock samples by an X-ray fluorescence spectrometer, (I) major elements (Abstract). Annual Meeting of Geological Society of China, pp. 418-420 (in Chinese).

Li, H.Q., Lu, Y.F., Wang, D.H., Chen, Y.C., Yang, H.M., Guo, J., Xie, C.F., Mei, Y.P., Ma, L.Y., 2006. Dating of the rock-forming and oreforming ages and their geological significance in the Furong Ore-field, Qitian Mountain, Hunan. Geological Review 52 (1), 113-121 (in Chinese with English abstract).

Li, X.H., 2000. Cretaceous magmatism and lithospheric extension in southeast China. Journal of Asian Earth Sciences 18, 293-305.

Li, X.H., Chen, Z.G., Liu, D.Y., Li, W.X., 2003. Jurassic gabbro-granitesyenite suites from Southern Jiangxi province, SE China: age, origin, and tectonic significance. International Geological Review 45, 898921.

Li, X.H., Chung, S.L., Zhou, H.W., Lo, C.H., Liu, Y., Chen, C.-H., 2004 Jurassic intraplate magmatism in southern Hunan-eastern Guangxi: ${ }^{40} \mathrm{Ar} /{ }^{36} \mathrm{Ar}$ dating, geochemistry, $\mathrm{Sr}-\mathrm{Nd}$ isotopes and implications for the tectonic evolution of SE China. In: Malpas, J., Fletcher, C., Ali, J.R. (Eds.), Aspects of tectonic evolution of China. Geological Society of London Special Publication 226, pp. 193-216.

Li, X.H., Li, Z.X., Li, W.X., Liu, Y., Yuan, C., Wei, G.J., Qi, C.S., 2007. $\mathrm{U}-\mathrm{Pb}$ zircon, geochemical and $\mathrm{Sr}-\mathrm{Nd}-\mathrm{Hf}$ isotopic constraints on age and origin of Jurassic I- and A-type granites from central Guangdong, SE China: a major igneous event in response to foundering of a subducted flat-slab? Lithos 96, 186-204.

Li, X.H., Zhao, Z., Gui, X., Yu, J., 1991. Sm-Nd and zircon U-Pb isotope constraints on the formation age of the Precambrian curst. Geochimica 3, 255-264 (in Chinese with English abstract).

Li, Z.X., Li, X.H., 2007. Formation of the 1300-km-wide intracontinental orogen and postorogenic magmatic province in Mesozoic South China: a flat-slab subduction model. Geology 35, 179-182.

Liu, C.S., Chen, X.M., Wang, R.C., Zhang, A.C., Hu, H., 2005. The products of partial melting of the lower crust: origin of Early Yanshanian Lapu Monzogranite, Guangdong province. Geological Journal of China Universities 11, 343-357 (in Chinese with English abstract).

Liu, Y., Liu, H.C., Li, X.H., 1996. Simultaneous and precise determination of 40 trace elements in rock samples by ICP-MS. Geochimica 25, 552-558 (in Chinese with English abstract).

Mahood, G., Hildreth, W., 1983. Large partition coefficients for trace elements in high-silica rhyolites. Geochimica et Cosmochimica Acta 47, 11-30.

Martin, H., Bonin, B., Capdevila, R., Jahn, B.M., Lameyre, J., Wang, Y., 1994. The Kuiqi peralkaline granitic complex (SE China): petrology and geochemistry. Journal of Petrology 35, 983-1015. 
Montel, J.M., 1986. Experimental determination of the solubility of Cemonazite in $\mathrm{SiO}_{2}-\mathrm{Al}_{2} \mathrm{O}_{3}-\mathrm{K}_{2} \mathrm{O}-\mathrm{Na}_{2} \mathrm{O}$ melts at $800{ }^{\circ} \mathrm{C}, 2 \mathrm{kbar}$ under $\mathrm{H}_{2} \mathrm{O}$-saturated conditions. Geology 14, 659-662.

Montel, J.M., 1993. A model for monazite/melt equilibrium and application to the generation of granitic magmas. Chemical Geology 110, 127-146.

Montel, J.M., Foret, S., Veschambre, M., Nicollet, C., Provost, A., 1996. Electron microprobe dating of monazite. Chemical Geology 131, 37-53.

Mordberg, L.E., Antonov, A.V., Petrov, O.V., Klindukhov, V.P., Kapitonov, I.N., Malich, K.N., Petrov, E.O., Sergeev, S.A., 2006. REE distribution as a tool for understanding origin of the Norilsk-1 differentiated ore intrusion. Geophysical Research Abstracts 8, 07349.

Nash, W.P., 1984. Phosphate minerals in terrestrial igneous and metamorphic rocks. In: Nriagu, J.O., Moore, P.B. (Eds.), Phosphate Minerals. Springer-Verlag, Berlin.

Pearce, J.A., Harris, N.B.W., Tindle, A.G., 1984. Trace element discrimination diagrams for the tectonic interpretation of granitic rocks. Journal of Petrology 25, 956-983.

Pearce, N.J.G., Perkins, W.T., Westgate, J.A., Gorton, M.P., Jackson, S.E., Neal, C.R., Chenery, S.P., 1997. A compilation of new and published major and trace element data for NIST SRM 610 and NIST SRM 612 glass reference materials. Geostandard Newsletter 21, 115 141.

Pirajno, F., Bagas, L., 2002. Gold and silver metallogeny of the South China Fold Belt: a consequence of multiple mineralizing events? Ore Geology Reviews 20, 109-126.

Sawka, W.N., Chappell, B.W., Kistler, W., 1990. Granitoid compositional zoning by side-wall boundary layer differentiation: evidence from the Palisade Crest intrusive suite, central Sierra Nevada, California. Journal of Petrology 31, 519-553.

Sha, L.K., Chappell, B.W., 1999. Apatite chemical composition, determined by electron microprobe and laser-ablation inductively coupled plasma mass spectrometry, as a probe into granite petrogenesis. Geochimica et Cosmochimica Acta 63, 3861-3881.

Shen, W.Z., Lin, H.F., 2002. Isotope studies of basement metamorphic, granitic and volcanic rocks in SE China and its crust evolution. In:
Wang, D., Zhou, X. (Eds.), Genesis of Late Mesozoic Granitic Volcanic-Plutonic Complexes in SE China and Crustal Evolution. Science Press, Beijing, pp. 230-272 (in Chinese).

Streckeisen, A.L., 1976. To each plutonic rock its proper name. Earth Science Review 12, 1-33.

Sun, S.S., McDonough, W.F., 1989. Chemical and isotopic systematics of oceanic basalts: implications for mantle composition and processes. In: Saunders, A.D., Norry, M.J. (Eds.), Magmatism in the Ocean Basins. Geological Society of London Special Publication 42, pp. 528-548.

Sun, T., 2006. A new map showing the distribution of granites in South China and its explanatory notes. Geological Bulletin of China 25, 332335 (in Chinese with English abstract).

Treloar, P.J., Colley, H., 1996. Variations in $\mathrm{F}$ and $\mathrm{Cl}$ contents in apatites from magnetite-apatite ores in northern Chile, and their ore-genetic implications. Mineralogical Magazine 60, 285-301.

Wang, H., Mo, X., 1995. An outline of the tectonic evolution of China. Episodes 8, 6-16.

Ward, C.D., McArthur, J.M., Walsh, J.N., 1992. Rare earth element behavior during evolution and alteration of the Dartmoor granite, SE England. Journal of Petrology 33, 785-815.

Xu, X.S., O'Reilly, S.Y., Griffin, W.L., Deng, P., Pearson, N.J., 2005. Relict Proterozoic basement in the Nanling Mountains (SE China) and its tectonothermal overprinting. Tectonics $24,1-16$.

Xu, X.S., O'Reilly, S.Y., Griffin, W.L., Wang, X., Pearson, N.J., He, Z., 2007. The crust of Cathaysia: age, assembly and reworking of two terranes. Precambrian Research 158, 51-78.

Yurimoto, Y., Duke, E.F., Papike, J.J., Shearer, C.K., 1990. Are discontinuous chondrite-normalized REE patterns in pegmatitic granite systems the results of monazite fractionation? Geochimica et Cosmochimica Acta 54, 2141-2145.

Zhang, S.L., Wang, L.K., Zhu, W.F., Yang, W.J., 1985. Use of REE in apatites to distinguish the petrogeno-mineralization series of granitic rocks. Geochimica 1, 45-57.

Zhou, X.M., Li, W.X., 2000. Origin of Late Mesozoic igneous rocks in Southeastern China: implication for lithosphere subduction and underplating of mafic magmas. Tectonophysics 326, 269-287. 